Historic, Archive Document

Do not assume content reflects current scientific knowledge, policies, or practices. 



\section{MARKET GARDENERS}

PERFECTION OF QUALI'TY

with us is of the

FIRST IMPORTANCE
THE COST OF ITS PRODUCTION

is always a

SECONDARY CONSIDERATION
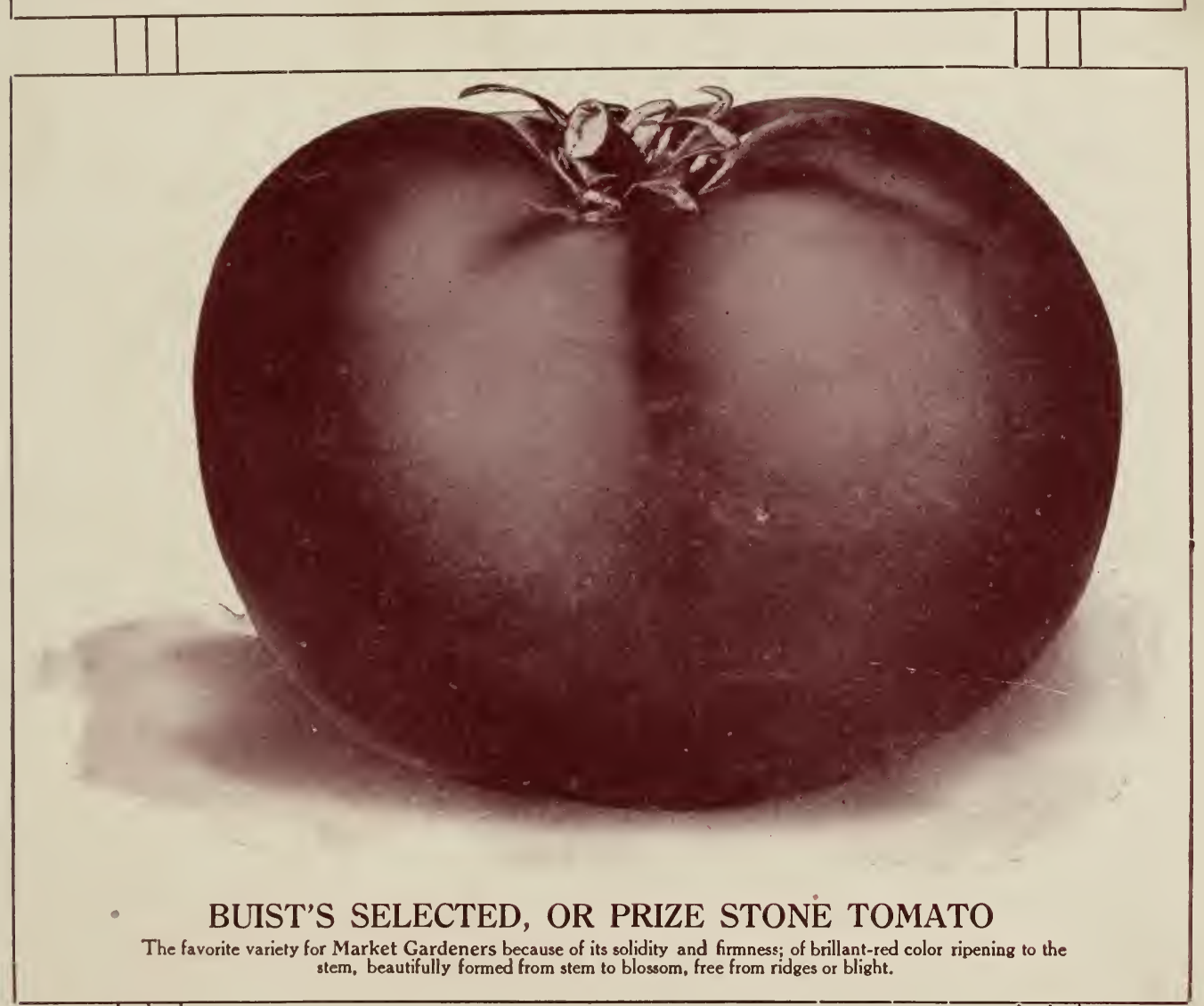

The favorite variety for Market Gardeners because of its solidity and firmness; of brillant-red color ripening to the stem, beautifully formed from stem to blossom, free from ridges or blight.

\section{ROBERT BUIST COMPANY SEED GROWERS}

Seed $\quad$ Nos. 4 and 6 South Front Street Warehouses $\left\{\begin{array}{r}\text { NoS. } \\ \text { 1st door below Market Street }\end{array}\right.$ 


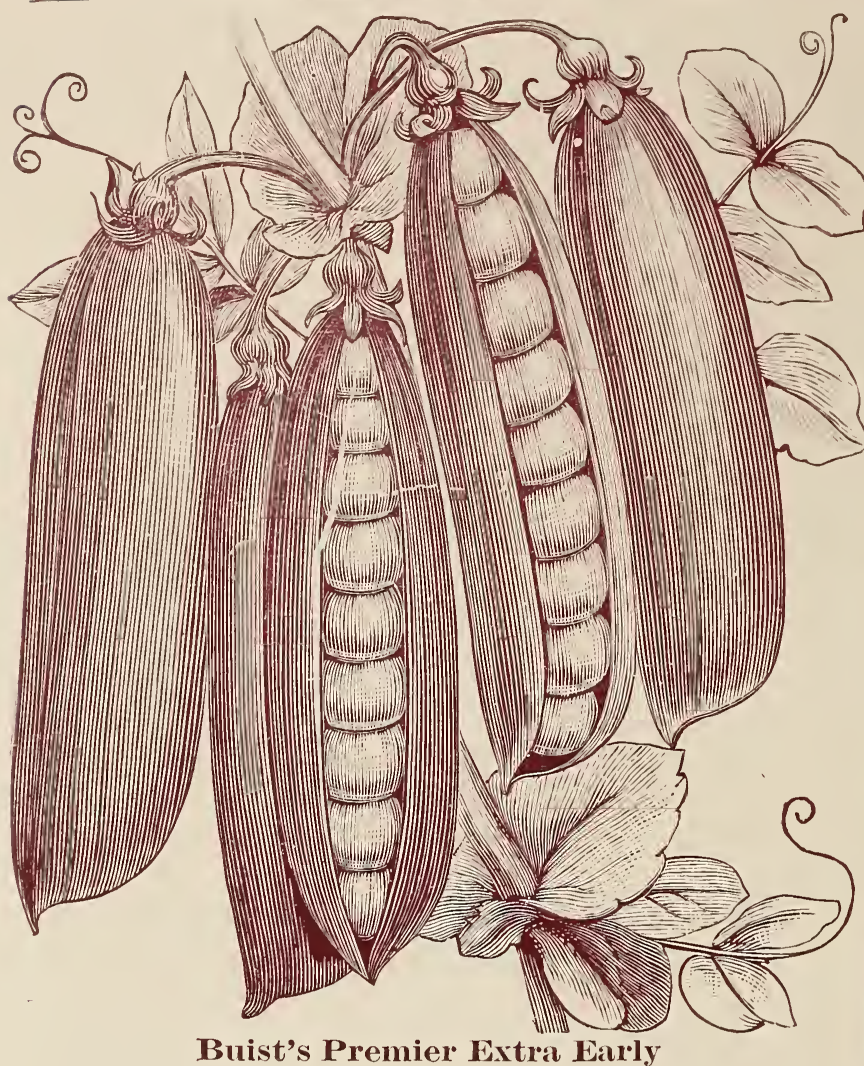

more largely by the market gardeners of this country than any other brand of Extra Earlies, except the Buist's Morning Star. Its productiveness, earliness, evenness of ripening and great delicacy of flavor are its great features. Price, $1 / 4$ Bushel, \$2.00; 1 Bushel, \$7.50.

Early Alaska. - This is the earliest blue variety in cultivation; the dark green color of its pods makes it a desirable shipping variety, as it will carry long distances without losing color; this quality combined with its extreme earliness recommends it very highly to the market garDener. Price, $1 / 4$ Bushel, $\$ 2.00 ; 1$ Bushel, $\$ 7.50: 10$ Bushels at $\$ 7.25$.

Extra Early Gradus, or Prosperity (Wrinkled).-This is the most famous of all the wrinkled varieties, and cannot be recommended too highly; it is the earliest wrinkled variety known; very hardy and a vigsrous grower, two and a half to three feet in height, very prolific and producing pods nearly as large as the famous Telephone, four to four and a half inches long, and filled with eight to ten large peas, which are of delicious flavor. Price, $1 / 4 \mathrm{Bushel}$, $\$ 4.25 ; 1$ Bushel, $\$ 16.00$.

Thomas Laxton.-This variety is nearly a; famous as the celebrated Gradus, which it closely resembles, produc ing very large, well-filled pods, with deep green-colored peas; it is very productive, sweet and delicious flavor, highlv recommended. Price, $1 / 4$ Bushel, $\$ 4.25 ; 1$ Bushel, $\$ 16.00$.

The Pea Crop this season is almost an entire failure, it is the shortest crop for the past 20 years.
BUIST'S PREMIER EXTRA

EARLY PEA

The earliest, Most Productive and

Most Profitable Variety for

Market (excepting the

Buist's Celebrated Morning Star)

Before the introduction of our famous Morning Star, this was the earliest and most profitable variety known, and today requires no comment from us to recommend it, as it is grown

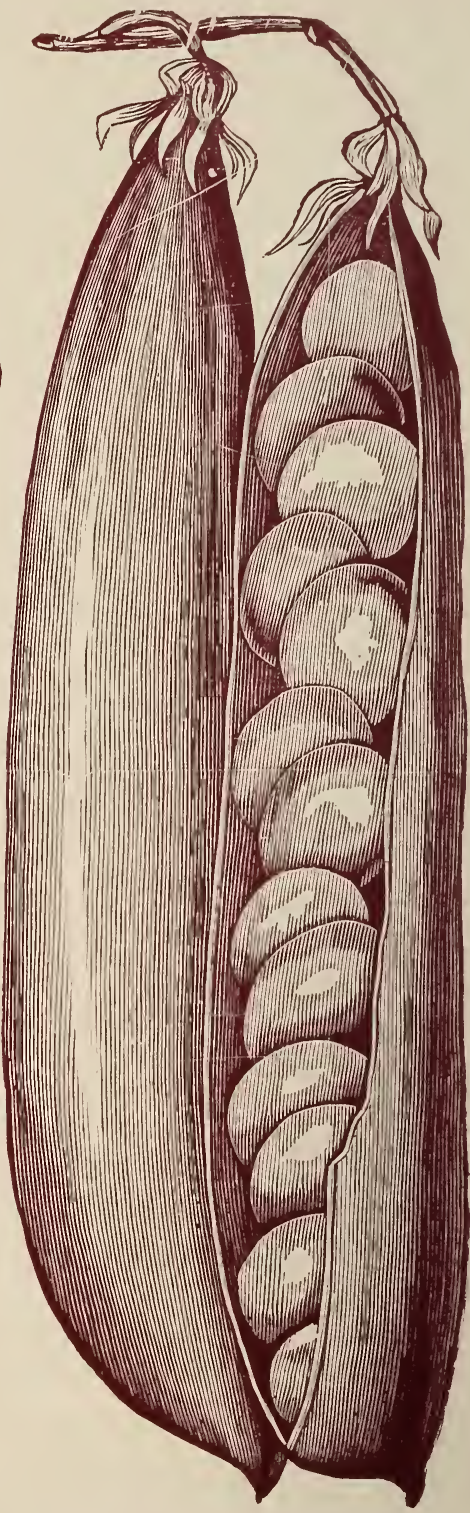

Gradus or Prosperity 


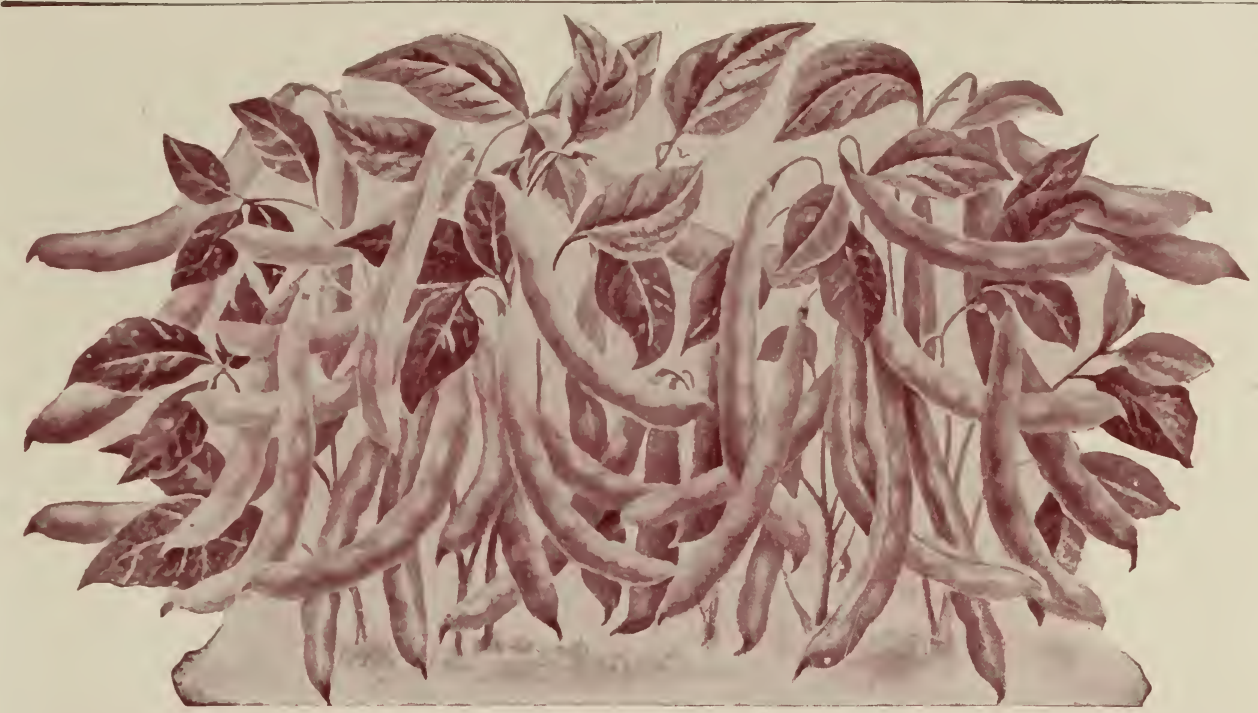

Buist's Lightuing Early Valentine Bean. Earlier than any other Valentine.

Buist's Lightning Early Valentine.-We recently introduced this variety which has become famous in all the Bean-growing sections of our country. It is well named as it is lightning in ripening in comparison with any other stock of this popular Bean. The pods are large, round and tender, is very productive, and when young entirely stringless. It cannot be commended too highly to the market-gardener, as he derives his greatest profit from the earliest crops, and this surpasses all other stocks of Valentines in both earliness and productiveness. It is sold only in our sealed packages and lead sealed sacks. Price, $1 / 4$ Bushel, $\$ 1.50 ; 1$ Bushel, $\$ 5.25$, 10 Bushels at $\$ 5.00$ per Bushel.

Black Valentine. - A recently introduced variety which has become quite popular in some localities, producing long. straight pods, which are quite attractive in their appearance. Price, 1/4 Bushel, $\$ 1.40 ; 1$ Bushel, $\$ 5.00 ; 10$ Bushels at $\$ 4.75$ per Bushel.

Burpee's Stringless Green Pod.-One of the finest of the stringless varieties, and now ranking with the famous Valentine in popularity and value. It cannot be recommended too highly to either the market gardener, private grower or canner; it is ready for the table as soon as the Valentine, and the pods being round and full, are very fleshly and succulent; they are abso-

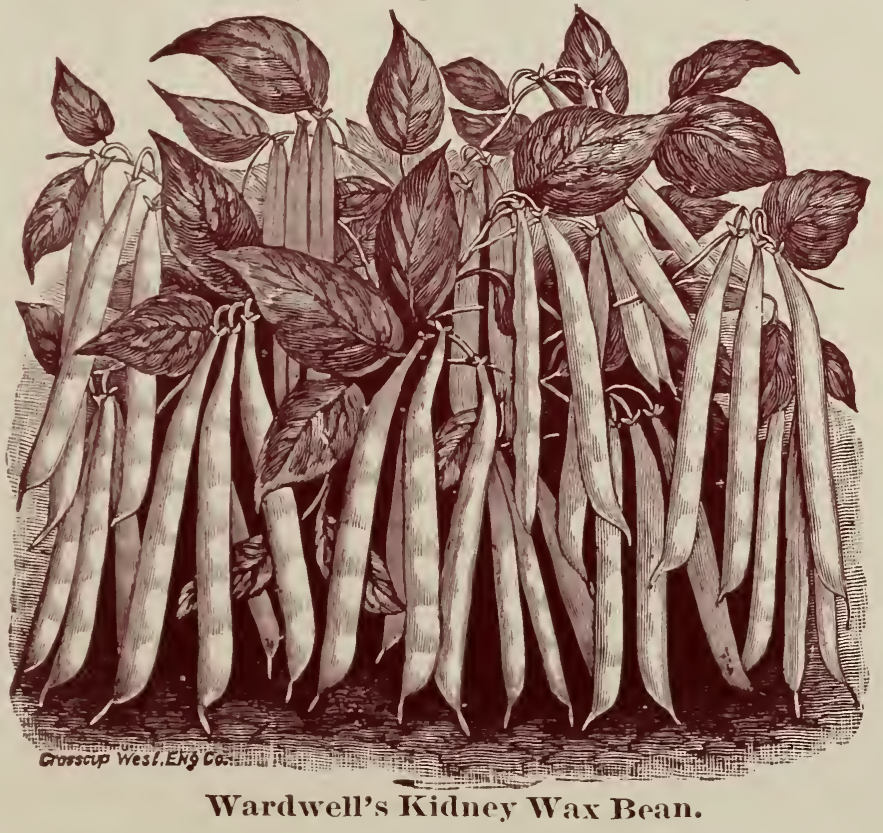
utely stringless, which secures for it the recommendation of every housekeeper, as there is not a more disagreeable dish for the table than stringy beans; aithough cooks profess to string them before using, yet they rarely do it. Price, $1_{4}$ Bushel, \$1.65; 1 Bushel, $\$ 5.75 ; 10$ Bushels at $\$ 5.50$ per Bush.

Wardwell's Kidney Wax. One of the most profitable varieties for the market gardener; especially popular in the South; long, straight,cream yellow pods of excellant quality; plant medium sized, maturing a little later than Golden Wax. This splendid variety is now a popular favorite everywhere, not only for shipping, but for the iocal market and the home. Price 1/4 Bushel, \$1.65; 1 Bushel, $\$ 5.75$; 10 Bushels at $\$ 5.50$ per Bushel. 


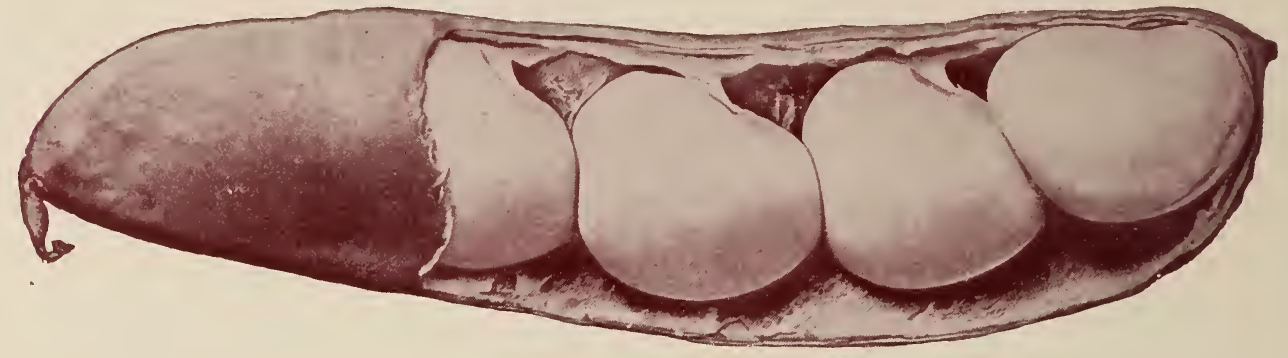

Fordhook Bush Lima

Fordhook Bush Lima.-A great improvement over the Dreer Bush, being more erect in its growth, more productive and producing larger pods, containing more beans, which, when even of full size, will cook tender, and of delicious flavor. Price, $1 / 4$ Bushel, $\$ 2.10 ; 1$ Bushel, $\$ 7.75$.

Burpee's Dwarf, or Bush Lima.-Produces beans as large as the Pole Lima, but in every other

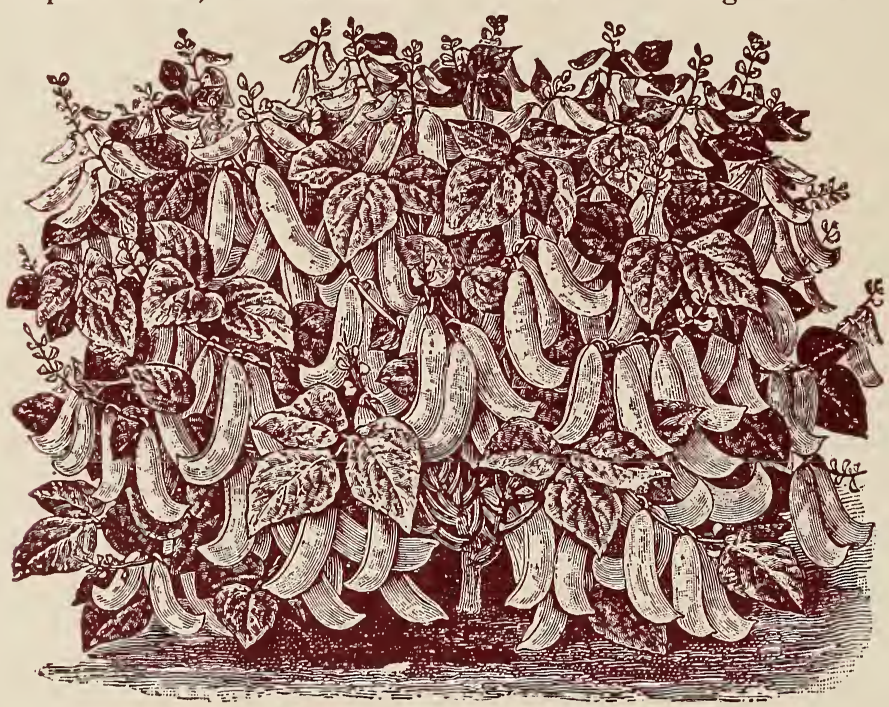
respect they are similar to the Henderson, except in quality, which we regard as superior, having more of the flavor of the old Lima; they are, however, more liable to contain runners; the plants grow about twenty inches high, of stout growth and erect, yet branching into a bush from two to three feet in diameter. Price, $1 / 4$ Bushel, \$2.00 ; 1 Bushel, $\$ 7.50$.

Burpee's Im proved Bush Lima.-Also an improvement over the popular Burpee's Bush, producing both pods and beans of a much larger size and two weeks earlier. Price, 1/4 Bushel, \$2.25; 1 Bushel,

\section{Burpee's Improved Bush Lima}

Dreer's Bush.-Possesses all the good qualities of the Dreer's Improved or Challenger Pole Lima, growing vigorously about two feet high and showing no disposition whatever to run. It produces pods in great abundance. The beans grow close together, four and sometimes five in a pod, and are thick, sweet and succulent. Price, $1 / 4$ Bushel, \$1.75; 1 Bushel, $\$ 6.50$.

Henderson's Dwarf, or Bush Lima.Many prefer the Dwarf Lima to the Pole Lima, yet in our estimation we regard the running variety decidedly p. -ferable, but the former has some advantages, as they require no support and come into bearing a month earlier. They grow fifteen to eighteen inches high and continue producing until frost. Price, $1 / 4$ Bushel, $\$ 1.75 ; 1$ Bushel, \$6.25.

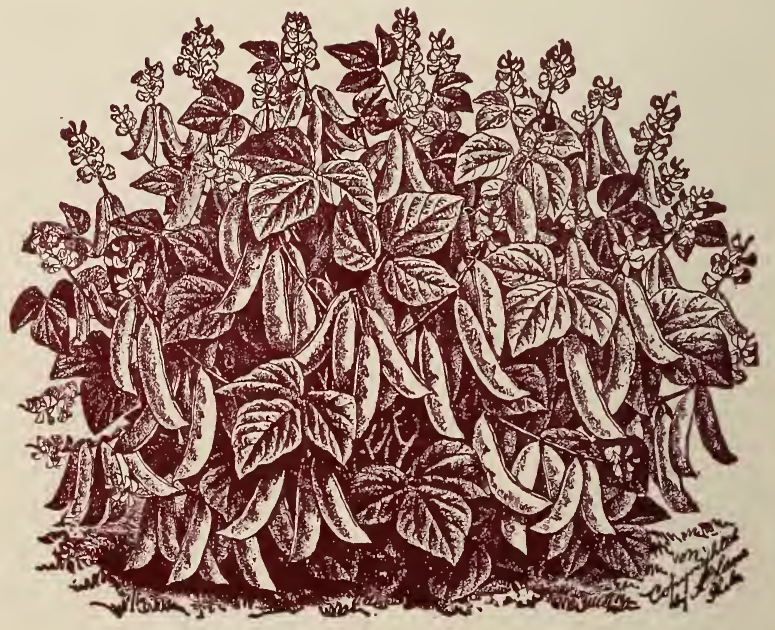

Dreer's Bush Lima 

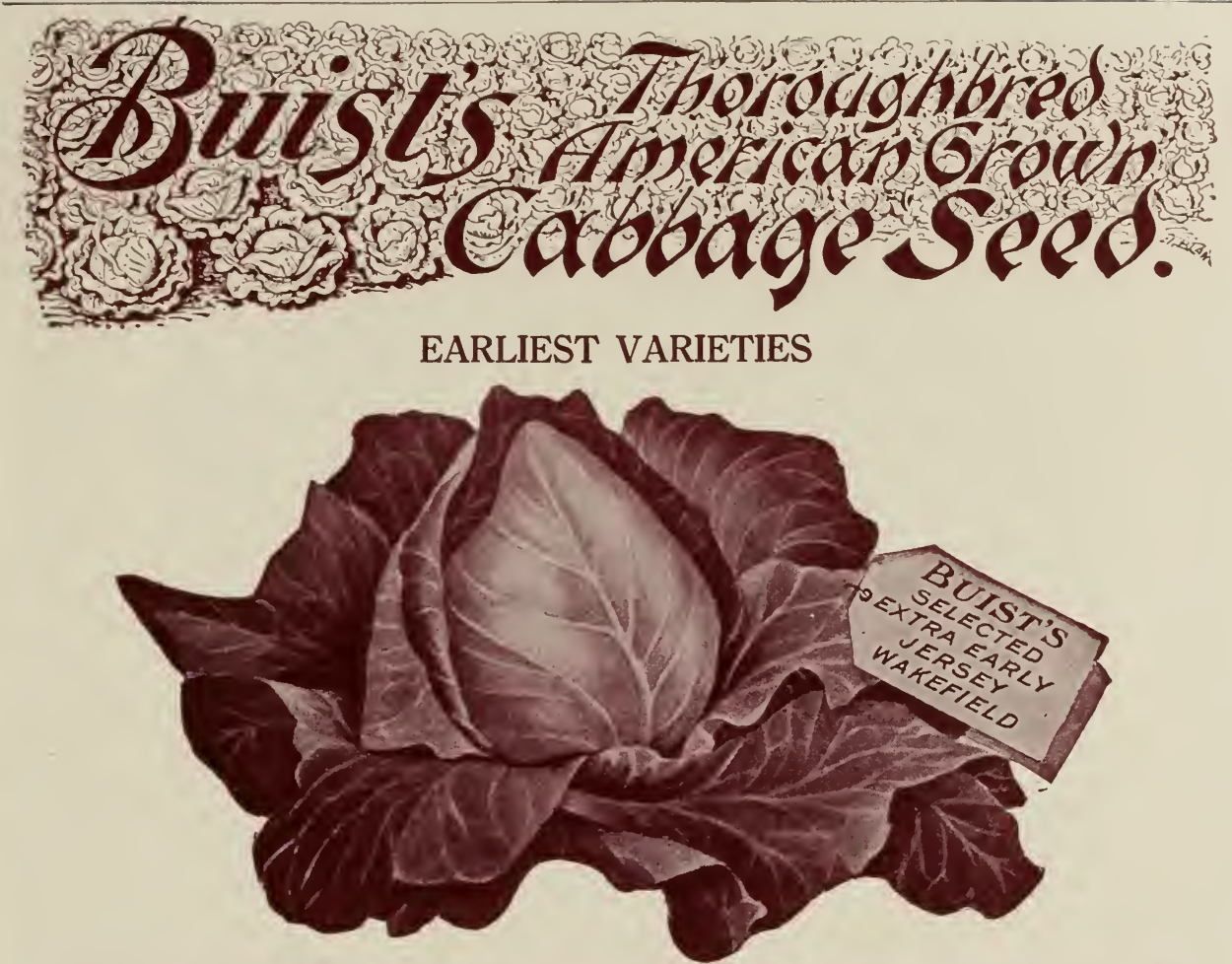

Buist's Selected Strain. (Its great Features are its Size and Earliness)

\section{THIS STOCK OF JERSEY WAKEFIELD IS UNSURPASSED}

\section{It is Sold only in our Sealed Cartons}

Early Jersey Wakefield (Buist's Selected Strain).-E Each year this variety is becoming more popular, and demand greater. It has not been very long since its growth was confined almost exclusively to the market gardeners of New Jersey, but it has gradually disseminated into other sections, and now is well known and highly appreciated by all the leading market gardeners of this country. It is the best and most profitable early variety to grow for either market or private gardens, as it is always sure to form fine, solid heads, and is the largest heading of all the early varieties. This special stock or strain is famous everywhere, both with market gardeners and private growers. It is sold only under our seal. There is no stock superior to it. Oz., 25c.; 1/4 lb., 75c.; lb., \$2.50.

The Charleston, or Large Jersey Wakefield. -This famous variety has all the fine qualities of the Early Jersey Wakefield; it produces heads of a much larger size, but is two weeks later. It has become a leading niarket variety in all sections of our country where the Cabbage is grown extensively for market. Oz., 25c.; $1 / 4 \mathrm{lb} ., 65 \mathrm{c}$. lb., $\$ 2.25$.

Buist s Earliest Flat Head.-This variety has given such great satisfaction that we consider it peerless anong the early sorts. Combined with earliness it unites compactness with excellent quality; forming flattish heads, and quite large for an early strain; having a short stem and but few outside leaves, permits of close culture. Oz., 25c.; 1/4 lb., 65c.; lb., $\$ 2.25$.

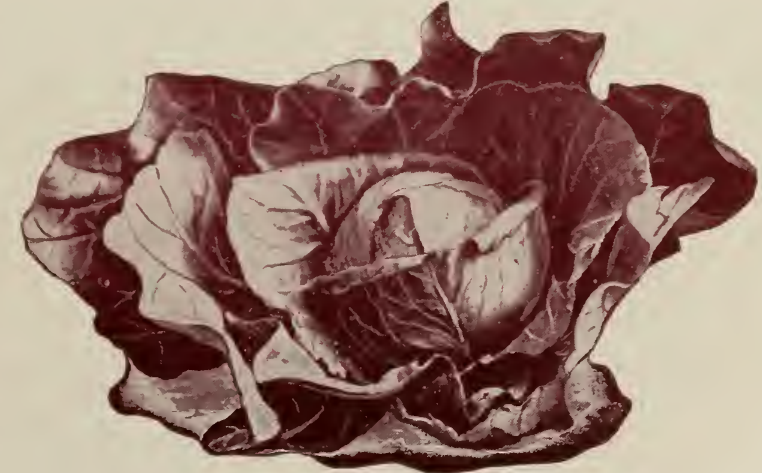

Buist's Earliest Flat-Head Cabbage 


\section{LATE VARIETIES OF CABBAGE FOR AUTUMN AND WINTER}

FORMING LARGE, SOLID HEADS

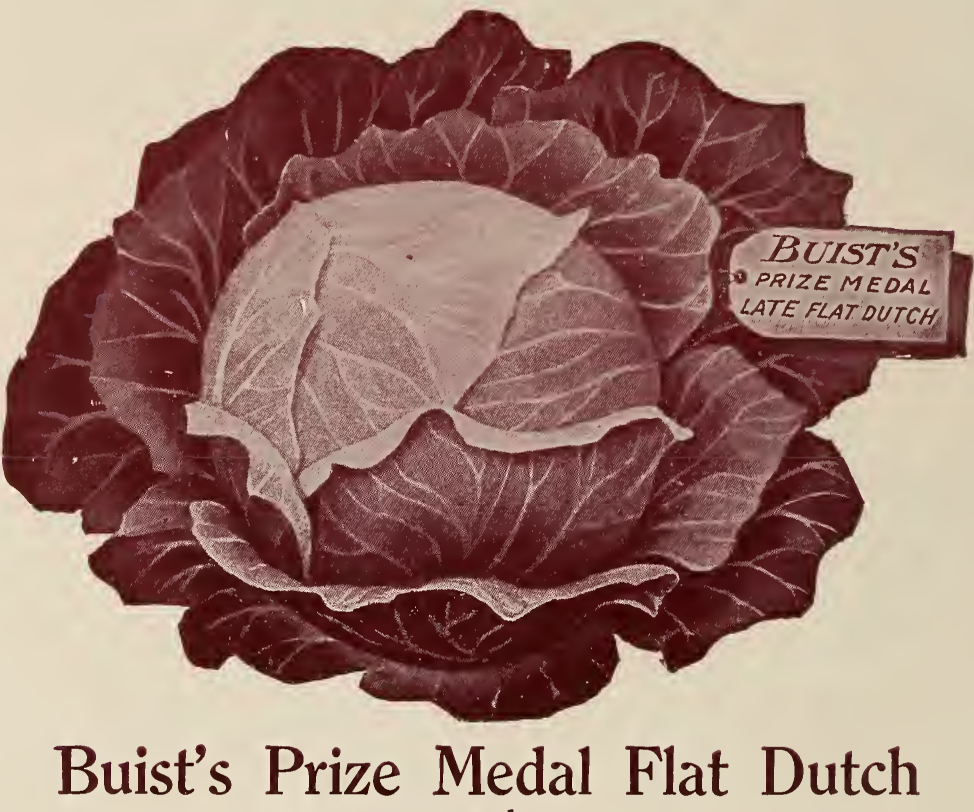

and

\section{Buist's Prize Medal Drumhead Cabbage}

Will Produce Large Solid Heads, in any Country and in any Soil. The richer the Soil the finer the Heads.

The Seed of this Cabbage is Sold only under our Seal, in Sealed Packets-Ounces, Quarters, Halves and Pounds. The Great Demand for it Requires us to Protect Ourselves and Customers in this Manner. Seed offered in any other way is Spurious Stock. Oz., 20c. ; $1 / 4$ lb., 65c. ; lb., \$2.25.

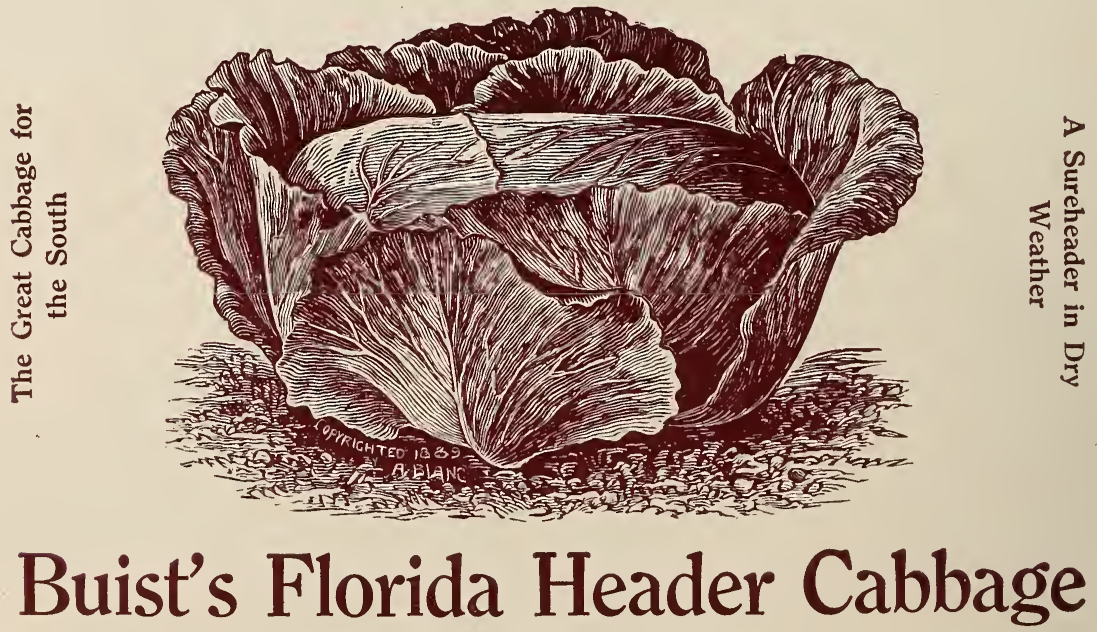

(Produces Heads from 15 to 30 pounds each)

The Seed of this variety is Sold only under our Seal, in Sealed Packets, Ounces, Quarters, Halves and Pounds. The Great Demand for it requires us to protect Ourselves and Customers in this manner. Seed offered in any other way is Spurious Stock.

$$
\text { Oz., 20c. ; } 4 \text { oz., 65c. ; lb., \$2.25. }
$$

See Pages 38, 40, 41 Buist's Catalog for description of above varieties. 


\section{BUIST'S PERFECTION EARLY WHITE SPINE CUCUMBER}
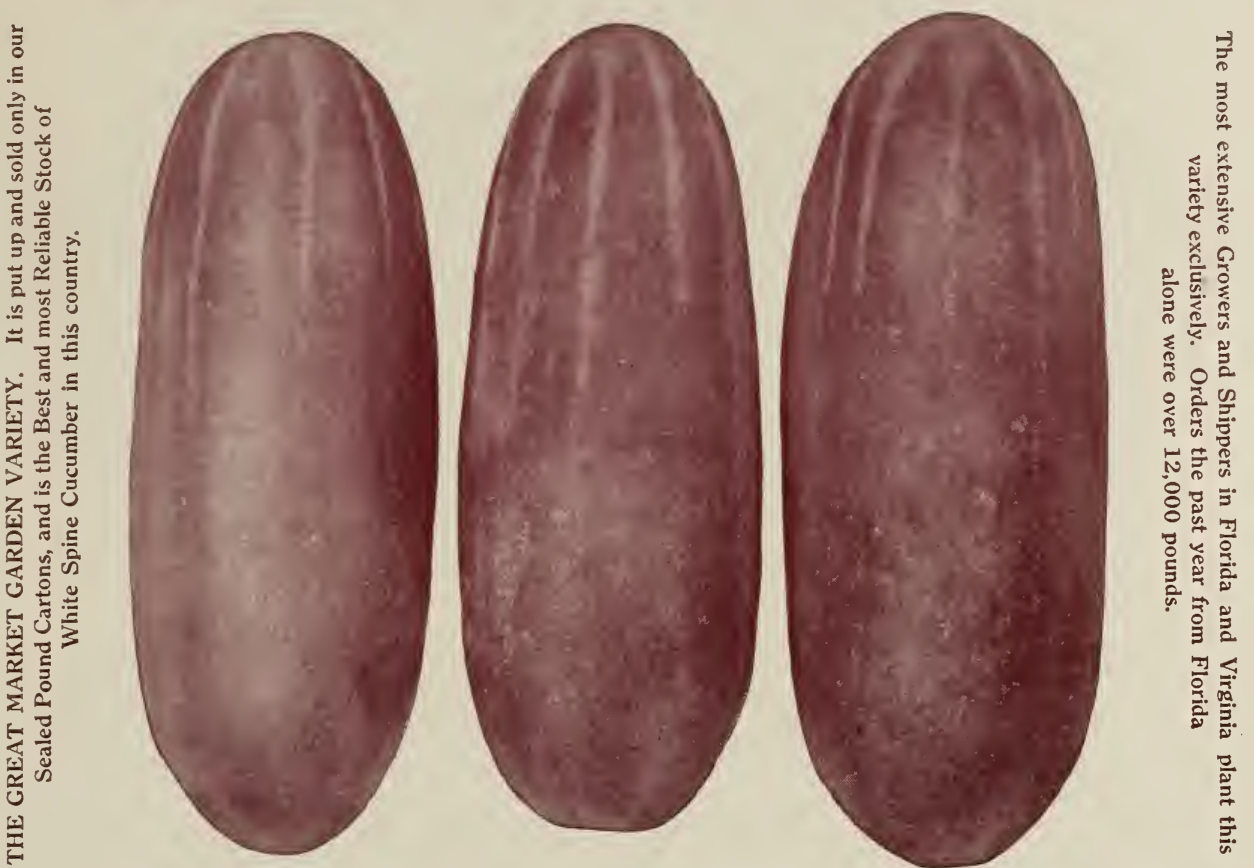

The Earliest, Finest Formed, Most Productive and Most Salable Variety to Grow for Market or Shipping. Price, $1 / 4$ lb., 35c. ; lb., $\$ 1.25 ; 5$ lbs. at $\$ 1.15 ; 10$ lbs. at $\$ 1.10$.

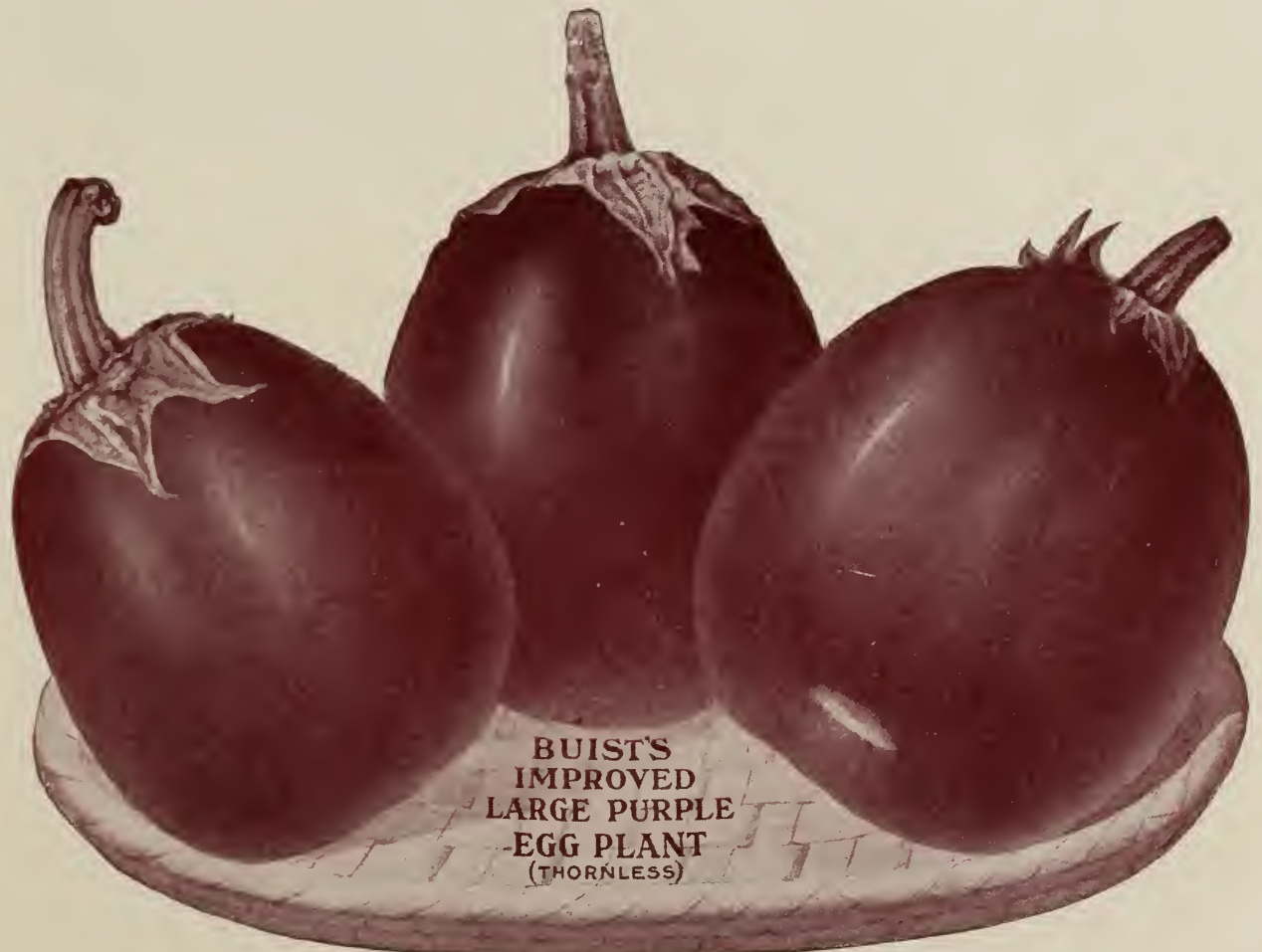

Perfect in Form, Very Large, Deep Purple and Spineless. 


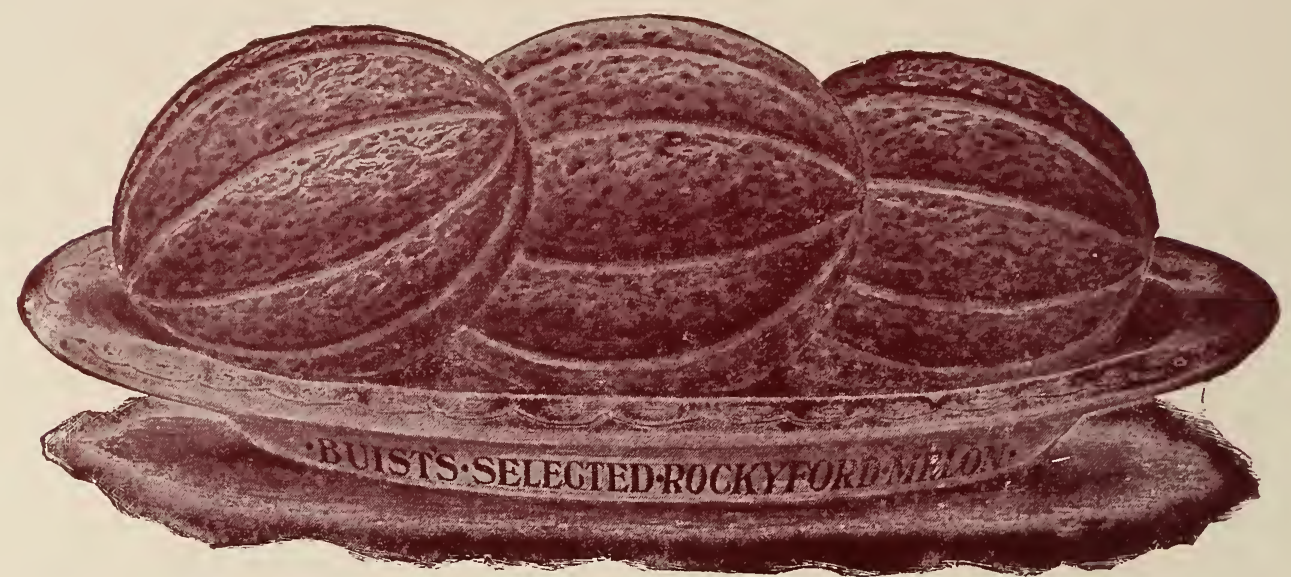

Rocky Ford (Buist's Selected).-A selection from the famous Netted Gem, introduced by the Rocky Ford growers of Colorado. It has become a famous shipping variety, is beautifully netted, of oblong shape, flesh deep green, and of superior quality. This variety differs from the Perfection in its form and thickness of flesh, and its silver netting does not cover the Melon so thoroughly. So popular has this name become that many of the leading fruit stores display any melons they may have. no matter how inferior they may be, under the name of Rocky Ford. Oz., 10c. ; $1 / 4$ lb., 25c. ; lb., 75c.

Burrell's Gem. - A very popular variety of the salmon flesh class, of medium size, oblong, well ribbed and covered with finely divided grayish netting, green skin, sweet and delicious; a very desirable market variety Oz., 10c. ; 1/4 lb., 25c.; lb., 80c.

Burpee's Fordhook.-This is without question the best of all the yellow-fleshed Cantaloupes, which are becoming so very popular in many localities; is about the same size as the Improved Jenny

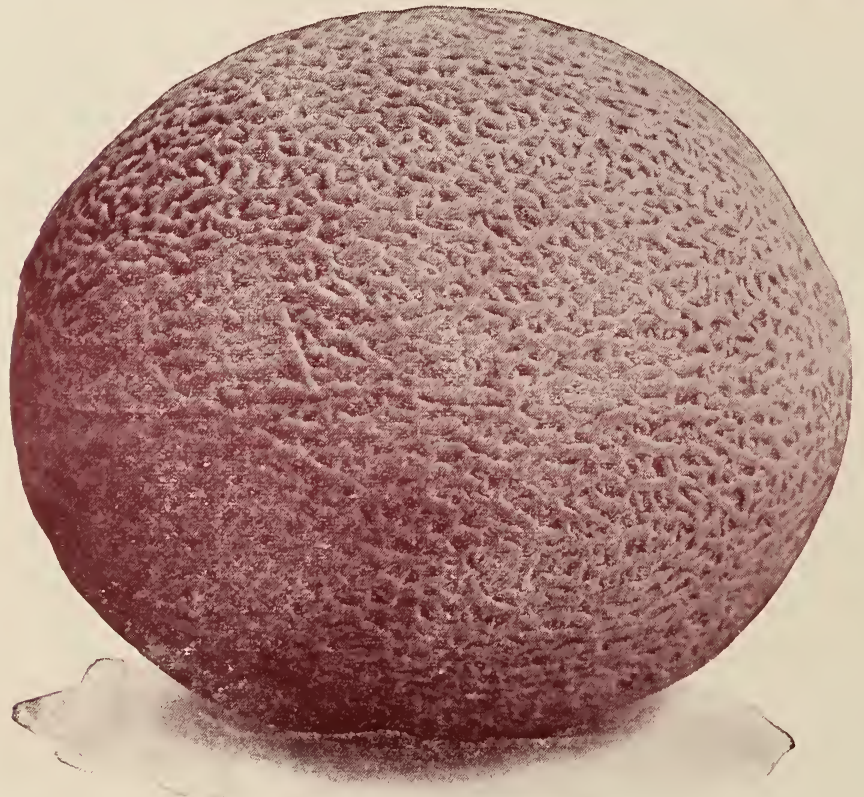

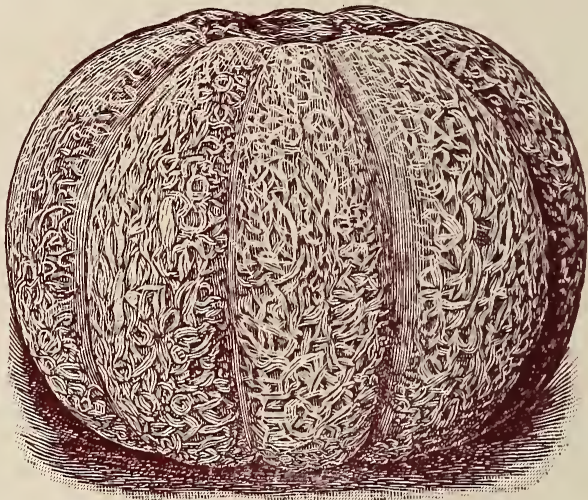

Fordhook Yellow Flesh Melon

Lind, heavily netted, flesh thick and solid, of a beautiful yellow color and of luscious flavor; seed cavity small average weight about two pounds; for market or private garden is unsurpassed and highly recomnlended Oz., 15c. ; 1'4 lb., 40c. ; lb., \$1.25.

Rocky Ford (Buist's Perfection . The Rocky Ford is the famous Netted Gem, introduced from Pennsylvania to Colorado, and in the dry atmospliere of the latter State attains extraordinary perfection so that it has become famous as a shipping variety and is now more generally known as the Rocky Ford Buist's Perfection is aspecially selected strain grown exclusively for us in Colorado and is absolutethe choicest stock obtainable; form almost round and completely netted, flesh green, very thick and of exqui-ite quality. Oz., 10c.; $1 / 4$ lb., 30c.; lb , $\$$ 


\section{THE THREE BEST WATERMELONS}

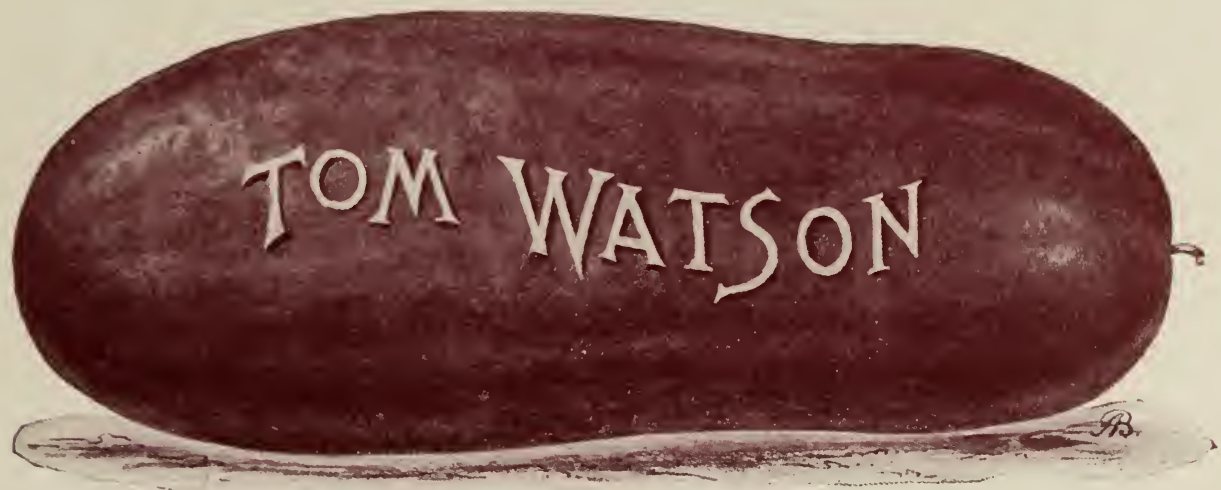

THE BEST' SHIPPER

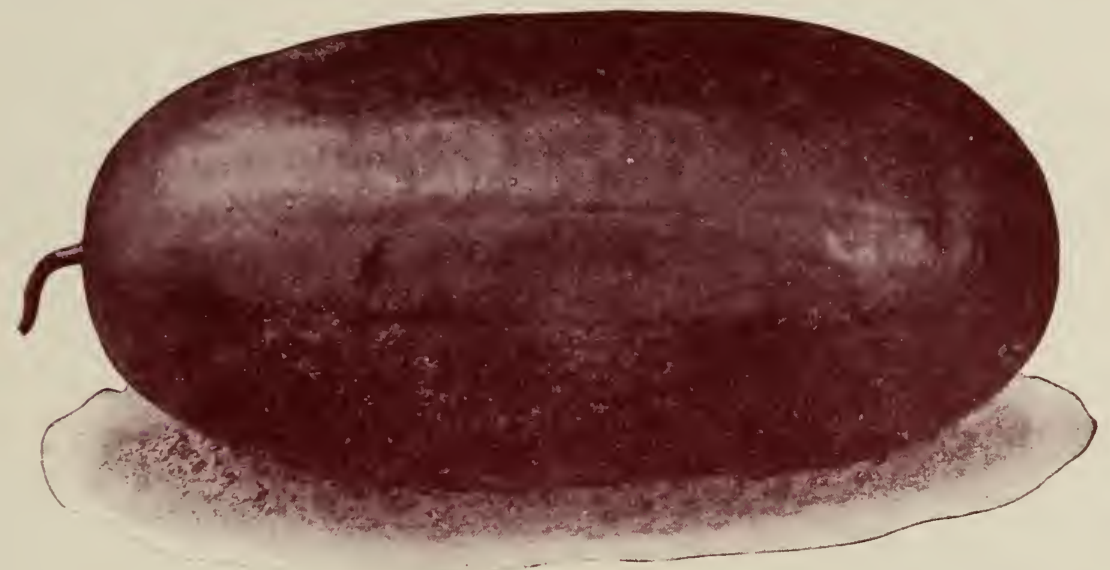

Kleckley's Sweet Watermelon “SWEETEST OF ALL” BEST FOR HOME USE

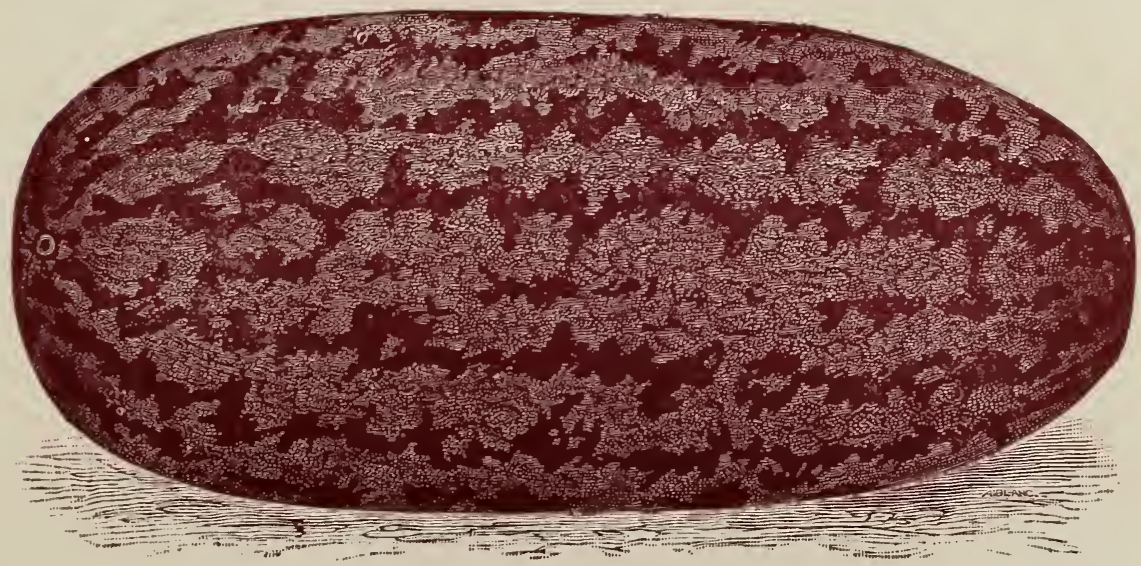

Florida Favorite Watermelon

THE BEST “ALL ROUND”

For description see Garden Guide Page 66 


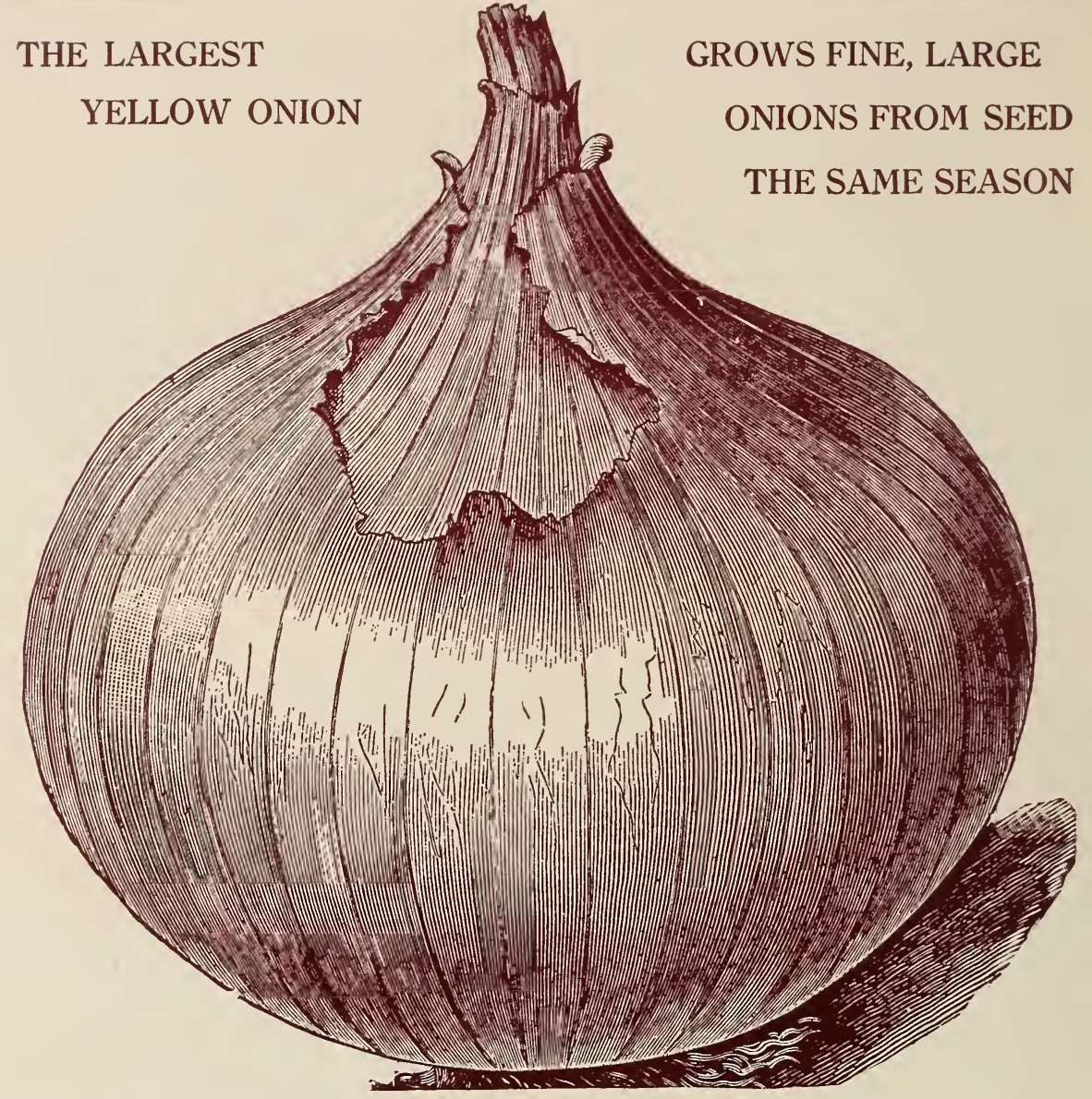

The Great Yellow Prize-Taker Onion-The Heaviest Cropper in the World.

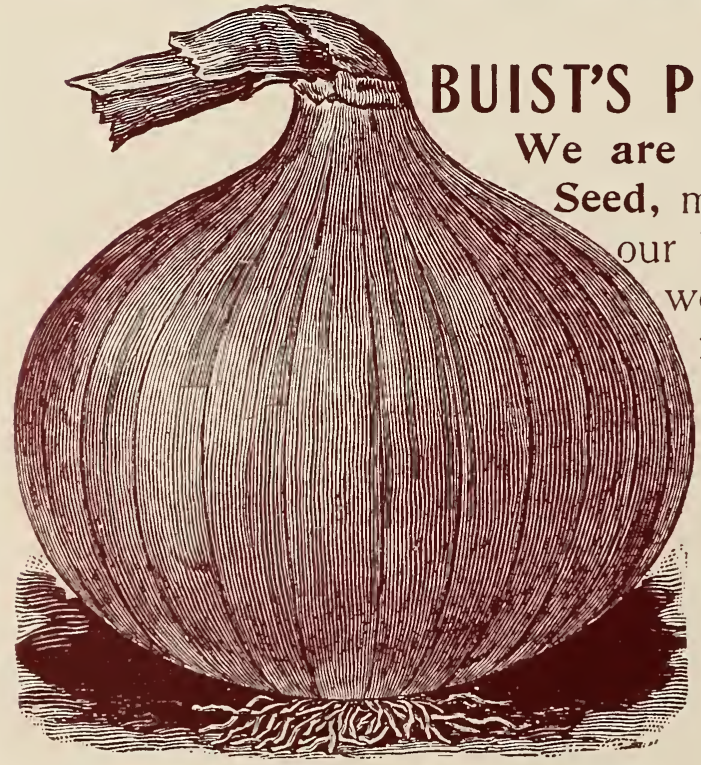

Buist's Prize Medal Globe Danvers Onion
PEDIGREE ONION SEED extensive growers of Onion aking it one of the specialties of House. The stock from which e grow our seed, is our old Pedigree stock, famous throughout the country for its purity and fine quality.

Buist's Yellow Globe $1 / 4$ b. per $1 \mathrm{~b}$, $1 \mathrm{bs}$. Danvers............. \$0 $40 \$ \$ 1.25 \$ 1.15$

Yellow Strasburg...... $\quad .35 \quad 110 \quad 100$

$\begin{array}{llll}\text { Large Red Wethersfield } & .40 & 1.25 & 1.15\end{array}$

White or Silver Skin.. $\quad .60 \quad 200 \quad 1.90$

$\begin{array}{lllll}\text { Southport Red Globe } & .40 & 1.30 & 1.20\end{array}$

$\begin{array}{lllll}\text { Southport Yel ow “ } & .40 & 1.20 & 1.10\end{array}$

Southport White “ $\quad \begin{array}{llll}75 & 2.25 & 2.15\end{array}$

Yellow I'rize-Taker.... $\quad .40 \quad 1.10 \quad 100$ 


\section{TOMATO}

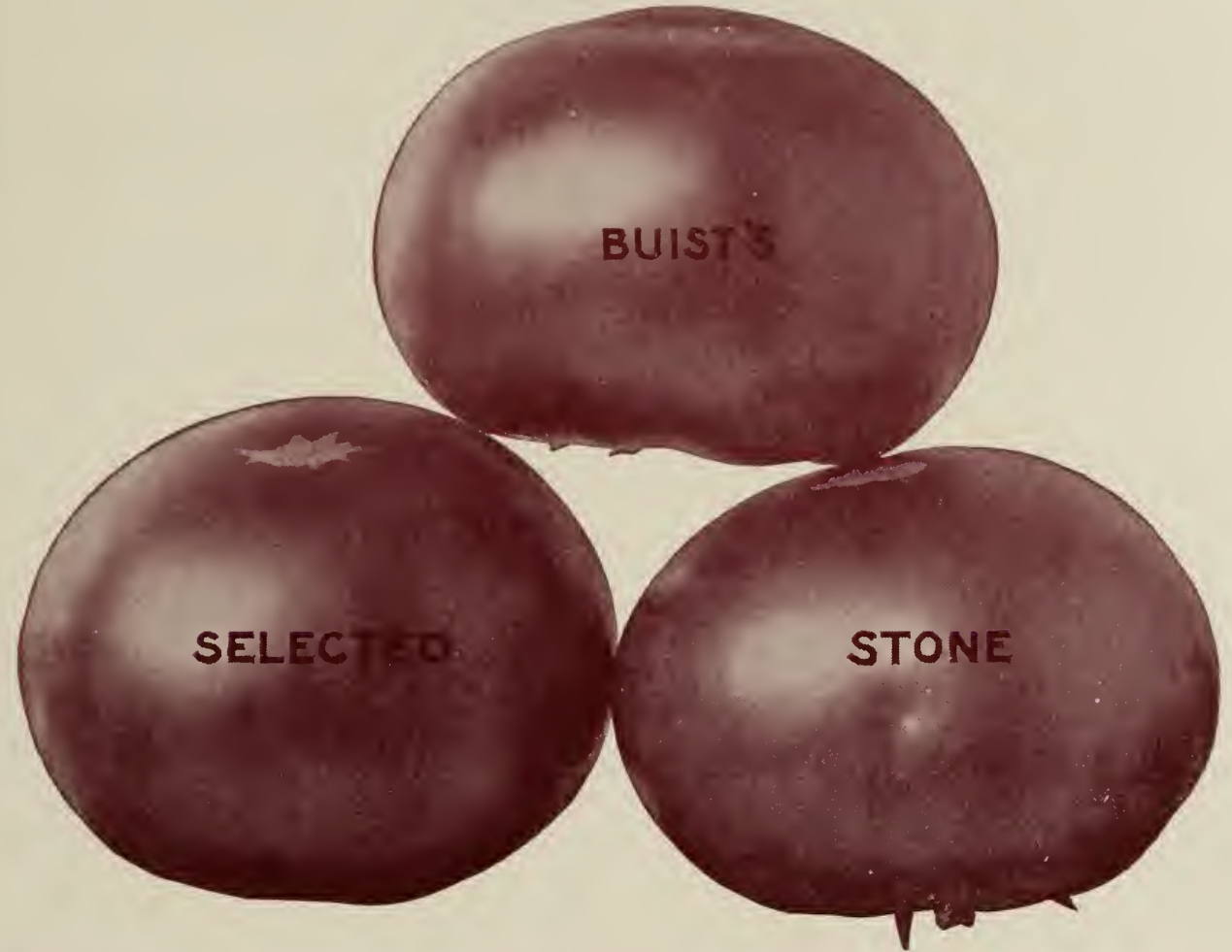

Buist's Selected, or Prize Stone.-The Stone is a great favorite with all cultivators of the Tomato, and this famous stock which we offer is the result of an annual selection for several seasons; it will be found a great improvement for its solidity, productiveness, form and richness of color, and is without question the finest stock for canners or for private gardens; highly recommended for a main crop, is of large size, bright scarlet color, very smooth, ripening evenly to the stem without a crack; exceedingly solid and firm-fleshed; is an excellent shipper; is a good keeper; free from core and not subject to rot; its appearance remarkably attractive; its vines and foliage robust, heavily loaded with very uniform specimens of fruit. $1 / 2 \mathrm{oz} ., 20 \mathrm{cts}$.; oz., 30 cts. ; 1/4 lb., 75 cts. ; lb., \$2.50.

Buist's New Ideal. - In introducing this new variety we are giving the Market Gardener a Tomato possessing all the desirable points of a PERFEC I' SPECIMEN, being very solid, of a most perfect sliade of scarlet, ripening uniformly close to the stem and has very few seeds, is free from crack or blemisli and grows to a very large size, besides is most productive and of very delicious flavor in addition to these superior traits its shipping qualities are exceptional, making it the most profitable market variety as well as the most desirable for private use. ${ }_{2}{ }_{2} \mathrm{Oz}, 35 \mathrm{c}$.; Oz., 50c. ; $1 / 4$ lb., $\$ 1.25$; lb., \$4.00.

Livingstone's Globe.-There is a constantly increasing demand for this variety from the largest tomato growing and shipping sections of Florida; its attractive globe shape, its beautiful purplish-pink color, productiveness and fine shipping qualities are its recommendation; besides, it is a good seller as an early market variety, $1 / 2$ oz., 20 cts. - oz., 30 cts. ; 1/4 lb., 85 cts. ; lb., \$3.00.

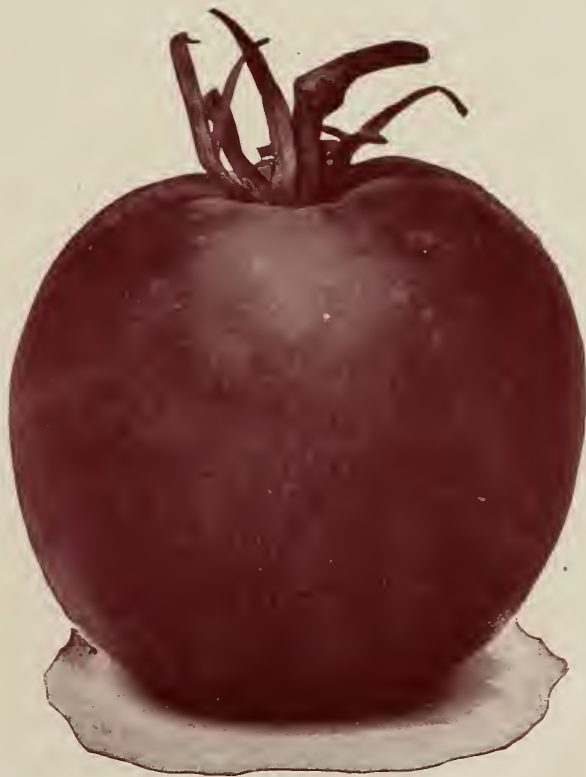

Livingstone's Globe 


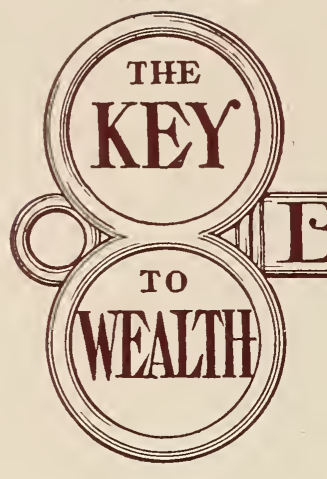

MARKET GARDENERS' WHOLESALE PRICE LIST

Buist's Seeds are grown from Selected Stocks and always of the highest quality.

BEANS-Green Podded

Improved Early Valentine. Buist's Lightning Valentine Black Valentine ......... Early Mohawk ...........

Early Yellow Six Weeks.. Extra Early Refugee......

Late Refugee .............

Stringless Green Pod......

Giant Stringless G'n Pod..

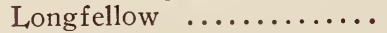

Bountiful $\ldots \ldots \ldots \ldots \ldots \ldots$

Dwarf Horticultural ......

\section{BUSH LIMA BEANS.}

Fordhook Bush Lima ..... I $25 \quad 2$ Io 7.75

Burpee's Dwarf Lima ..... I oo $1.75 \quad 650$

Burpee's Imp. Bush Lima.. I $25 \quad 2.00 \quad 7.50$

Henderønn'? Push Lima .. $\quad$ I.00 $\quad 1.75 \quad 6.25$

Dreer's Busn Lima........ $1.00 \quad 1.756 .50$

Dreer's Wonder Bush..... $1.00 \quad$ I.75 6.50

Wood's Bush Lima ....... I.00 1.756 .50

\section{BEANS - Wax or Golden Podded.}

Dwarf German Blk. Wax.. 1.00 I.65

Dwarf Pro'fic Ger. Wax.. I.oo I 655575

Dwarf Golden Wax....... I oo I 65575

Improved Golden Wax.... I oo I 655575

Wardwell's Kidney Wax... I oo $\quad$ I.65 575

Round Pod Kidney Wax.. $\quad$ I.oo I 756.50

Dwarf Wax Bismark...... I oo I.65 6 oo

Perfection Dwarf Wax.... I oo r.65 6 oo

Crystal White Wax ...... I oo I 75650

Davis White Wax ........ I oo 1.656 .00

Currie's Rustproof Wax... I.oo I $65 \quad 575$

Burpee's White Wax ..... I oo I 756.50 BEANS-Pole.

Large White Lima ....... I oo I 656.00

Salem Mammoth Lima.... I co I 75 6.50

Extra Early Jersey Lima.. I oo $\quad$ I.75 6.25

King of the Garden Lima.. I oo I 756.25

Ford's Mammoth Lima.... I.00 I 75650

Ideal Mammoth Lima .... I Io I 857 oo

Dreer's, or Challenger..... I.00 I 656.00

Carolina, or Sieva Lima...

Southern Crease-Back .... I I o I 656.00

German, or Black Wax.... I.oo I 75650

Lazy Wife's Pole ........ I.25

Golden Cluster Wax ...... I 252 oo 750

London Horticultural ..... I.00 I.65 600

Monster Southern Prolific. I.oo I 656.00 PRIZE SEEDS

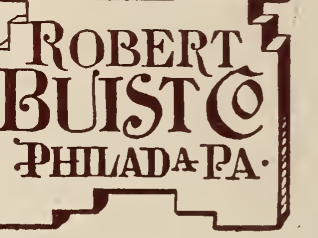

\section{BEANS-Pole-con't}

Dutch Case Knife ........

Kentucky Wonder $\ldots \ldots \ldots$ I 00 I.65 600

Kentucky Wonder ........ I o 1.656 .00
Buist's Early Morning Star I.25 2.258 .00 (Sealed Bags only.) This is the Earliest and Best Variety. Buist's Premier Ex. Early I.I5 $2.00 \quad 7.50$ Extra Early Market....... I.I5 $2.00 \quad 7.50$ Earliest of All ........... I.I5 $2.00 \quad 7.50$ First and Best ............. I. 1.25 2.00 750 Extra Early June ........ I I $25 \quad 2$ oo 7.50 Philadelphia Extra Early.. I.25 2.00 750 Alaska, or Blue Wonder.. I $252.00 \quad 7.50$ Early American Wonder. I 40250900 Nott's Excelsior .......... I I $40 \quad 250 \quad 9.00$ Extra Early Gradus ...... 225425 I6. o0 Thomas Laxton .......... $2.25 \quad 4.25 \quad 16.00$ McLean's Little Gem...... I. 1.502509 .00 Early Premium Gem...... $1.50250 \quad 9.00$ Sutton's Excelsior ......... I 753 oo II oo Imp'd Dan O'Rourke...... I I $25 \quad 2$ oo $7.5^{\circ}$ Early Washington ......... I.25 $200 \quad 750$ Early Frame .............. 1.25 $2.00 \quad 750$ Early May ................ I.25 2.00750 Early Tom Thumb........ Horsford's Market Garden $1.40 \quad 2.50 \quad 9.00$ Heroine ............... I.40 $\quad$ I.50 9.00 McLean's Advancer ...... I.40 $\quad$ I.50 9.00 Bliss's Abundance ........ $1.25 \quad 2.25 \quad 8.00$

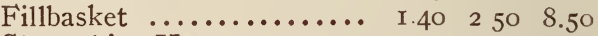
Shropshire Hero .......... $1.40 \quad 250 \quad 9.00$ Long Island Mammoth.... I.5O 275 I0.00 Duke of Albany ........... I 75 3 Oo II.oo Potlatch............ I $75 \quad 3.00$ II oO Boston Unrivaled ........ I.50 $2.75 \quad$ I0.00 Champion of England...... Yorkshire Hero .......... Pride of the Market ...... I. I. $40 \quad 2.50 \quad 9.00$ Bliss's Everbearing ........ I.25 $2.25 \quad 8.00$ Telephone ............... Improved Stratagem ...... I. $1.50 \quad 2.75$ 10.00 Eugenie ............... I.40 $\quad 2.50 \quad 9.00$ Dwarf Blue Imperial ..... I.I5 $2.00 \quad 7.00$ Large White Marrowfat... $\quad 85$ I.50 $\quad 5.00$ Black-Eyed Marrowfat... $\quad .85 \quad 1.50 \quad 5.00$ Dwarf and Tall Sugar..... I.50 $\quad 2.75$ I0.00 Mammoth Podded Sugar.. $1.75 \quad 3.25$ I2.00 Melting Sugar ............ I.75 3.25 I2.00 
ASPARAGUS.

Conover's Colossal

Barr's Mammoth

The Palmetto ............ .10

Giant Argenteuil

Columbia Mamith IVhite.

ASPARAGUS ROOTS.

Conover's Colossal .........

Barr's Mammoth ...........

The Palmetto ..............

Giant Argenteuil ..........

Columbia Nam'th White.

BEET - Turuip-Rooted Varieties. Per Per Per

Extra Early Bassano......

Buist's Ex. Early Turnip..

Ex. Early Egyptian Turnip.

Buist's Ex. Early Egypt'n.

Crosby's Early Egyptian..

Early Eclipse Turnip .....

Detroit Blood Turnip.....

Early Dark Blood Turnip.

Early Othello Blood Turnip

Bastian's Early Red Turnip

Dark Stinson .............

Crimson Globe ............

Edmand's Red Turnip.....

Swiss Chard ............

$\begin{array}{ccc}\text { Fer } & \text { Per } & \text { Per } \\ \text { oz. } & \text { 1.t } & \text { ib. } \\ \text { ib. } \\ .10 & .15 & .40 \\ .10 & .15 & .40 \\ .10 & .15 & .40 \\ .10 & .25 & .75 \\ .10 & .20 & .50\end{array}$

Per Per

I00 joco

$.50 \quad+.00$

$.50 \quad 4.00$

.504 .00

.605 .03

$.50 \quad 4.50$

$1 / 4$ 1b. $1 \mathrm{~b}$.

$30 \quad I 0 O$

.40 I 25

$.40 \quad$ I 25

$.45 \quad \mathrm{I} .5 \mathrm{O}$

45 I $5^{\circ}$

30 I OO

$.45 \quad \mathrm{I} .5^{\mathrm{O}}$

40 I 25

.45 I 50

$.40 \quad$ I 25

$.40 \quad$ I 25

.40 I 25

$.40 \quad$ I. 25

$.25 \quad .75$

BEET (Long Varieties).

$\begin{array}{llll}\text { Buist's Scarlet Perfection.. } & \text {. I5 } & .40 & \text { I.25 }\end{array}$

Bastian's Half Long...... .I5 .40 1.25

Long Blood ............. . . .

Buist's Imp. I.ong Blood.. .I5 .45 I 50

BEET - Stock-Feeding Mangels.

Buist's Prize Gold. Globe.

Chirk Castle Mammoth....

Orange Globe ............

Champion Prize L'g Red..

Golden Tankard ..........

Buist's Mam. Long Red...

Taber's Gate Post ........

Long Red ..............

White French Sugar ......

Klein Wanzelben Sugar...

BROCCOLI.

Early Purple Cape .......

Early Walcheren

.25

.30

.75

2.75

BRUSSELS SPROUTS.

Early Paris Market .......

Long Island Dwarf .......

.15

$.40 \quad 1.25$

$.20 \quad .65 \quad 2.25$

CABBAGE-Earliest varieties.

Buist's Jersey Wakefield..

Early Jersey Wakefield....

Charleston, Wakefield ....

Buist's Earliest Flat Head

Early Winnigstadt ........

Extra Early Express .....

Buist's Early Large York.

Extra Early Volga .......

Extra Early Eureka ......
CABBAGE - Early varieties

Per Per Per

Allhead Early

$\begin{array}{llll}.65 & 2 & 25\end{array}$

Henderson's Succession ... $\quad .20 \quad .65 \quad 2.25$

Buist's Early Drumhead... . .20 652525

Buist's Early Flat Dutch. $\quad .20 \quad .65 \quad 225$

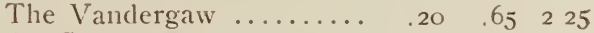

$\begin{array}{lllll}\text { All Seasons .............. } & .25 & .75 & 250\end{array}$

Fottler's Imp. Brunswick. $\quad .20 \quad .50 \quad$ 1.60

Buist's Florida Header... $\quad .20 \quad .65 \quad 2.25$

Green Glazed ........... . .20 $\quad .60 \quad 1.75$

Buist's Brunswick D'ml'd, $\quad .20 \quad .60 \quad$ I.75

CABBAGE-Late varieties.

$\begin{array}{llll}\text { Buist's Prize Med. Drumhead } \quad .20 & .65 & 2.25\end{array}$

Buist's Prize Medal Flat

Dutch ............... .20

Premium Late Drumh'd... .20

Premium Late Flat Dutch. $\quad .20$

Danish Ball-Head (Tall).

Ball-Head (Dwarf)

Large Surehead ..........

Autumn King ............

Louisville Drumhead ......

Stone Mason ............

Marblehead Mammoth ....

North Carolina Buncombe.

Red Dutch ............. .20

Mammoth Red Rock ....... .20

Blood Red Berlin ........ . .20

Blood Red Erfurt ...... . 20

$\begin{array}{ll}.65 & 2.25\end{array}$

$.50 \quad$ I. 50

$.50 \quad$ I. 50

$.90 \quad 300$

$90 \quad 3.00$

$.50 \quad 1.75$

$.50 \quad 1.75$

$.40 \quad 1.50$

$.40 \quad 1.50$

$.40 \quad 1.50$

$.60 \quad 1.75$

$.60 \quad 1.75$

$.60 \quad 1.75$

$.60 \quad 1.75$

$.60 \quad 1.75$

\section{CABBAGE, SAVOY.}

Green Curled Savoy...... . . I5 $\quad .50$ I.40

Green Globe Savoy ....... .I .50 I. 50

Large Drumhead Savoy... $\quad .15 \quad .50 \quad$ I.40

$\begin{array}{llll}\text { Perfection D'mh'd Savoy.. } & \text {. I5 } & .50 & \text { I.40 }\end{array}$

\section{CARROT.}

Early French Forcing..... .I5 .45 I 50

Early Scarlet Horn....... I5 45 I 50

Guerande, or Oxheart.... $\quad .15 \quad .45$ I 50

Buist's Half-Long Scarlet. . I5 .45 I 50

Half-Long Chantenay ..... . . $15 \quad .45$ I 50

Danver's Half Long ....... . I5 .45 I 50

St. Vallery Half-Long..... . . I5 .45 I 50

Half-Long Scarlet, Blunt.. . I5 45 I 50

Half-Long Carentan ...... I5 $\quad .45$ I 50

Buist's Long Orange........ I5 45 I $5^{\circ}$

Long Orange $. . . \ldots \ldots \ldots . . . .15 \quad .45$ I 50

\section{CAULIFLOWER.}

Buist's Early Snowball... $2.25 \quad 7.50 \quad 22.00$ Famous Dry Weather..... $2.75 \quad 8.00 \quad 25.00$ Extra Early Dwf. Erfurt.. $2.25 \quad 7.5022 .00$ Algiers ................. $\quad .65 \quad 1.85 \quad 7.00$

Early Dwarf Erfurt....... I.25 $\quad 4.50$ 16.00

Extra Early Paris......... .65 1.85 7.00

Half Early Paris......... .65 I.85 7.00

Le Normand Short Stem.. $\quad .50 \quad$ I.75 6.00

Veitch's Autumn Giant.... $\quad .40$ I.50 5.00 


\section{CELERY.}

Large White Solid........

Buist's Large White Solid.

Golden Self - Blanching (American) ............

Golden Self - Blanching (French) ...............

White Plume ...........

Giant White Pascal ......

Winter Queen ...........

Golden Dwarf ...........

Dwarf Golden Heart......

Golden Market ..........

Boston Market ...........

Celeriac ................

CORN-Garden Varieties.

Early Red Cory ..........

Mammoth White Cory ...

Early Marblehead ........

Golden Bantam ..........

Crosby's Early ...........

Early Minnesota ..........

Stabler's Early ...........

Early Cosmopolitan ......

Early Metropolitan .......

Shoe Peg ...............

Country Gentleman .......

Perry's Hybrid ...........

Kendall's Early Giant......

Potter's Excelsior ........

Early Triumph ...........

Hickok's Improved ........

Early Mammoth ........

Late Mammoth .........

Stowell's Evergreen ......

New England 8-Rowed....

Egyptian ...............

Black Mexican ..........

Adams' Extra Early ......

Adams' Early .............

Perkins' Early Market ....

\section{CORN-Field varieties.}

Buist's Golden Dent ......

Buist's Southern Snowflake.

Improved Early Leaming..

Early Golden Beauty .....

Cloud's Mammoth Dent....

Chester County Mam'oth..

White Cap Yellow Dent....

Hundred-Day Bristol .....

Penna. Eight-Rowed .....

Early Yellow Canada......

Mastodon Yellow Dent....

Early Northern White Dent

Trucker's Favorite .......

Maryland White Dent....

White Hickory King......

Large White Flint........

Blount's White Prolific...

Mam'oth White Surprise..

Champion White Pearl....

White Cob Ensilage......

Red Cob Ensilage .......

\begin{tabular}{|c|c|c|}
\hline $\begin{array}{l}\text { Per } \\
\text { Oz. }\end{array}$ & $\begin{array}{l}\text { Per } \\
x / 4 \text { lb. }\end{array}$ & $\begin{array}{l}\text { Per } \\
\text { lb. }\end{array}$ \\
\hline .15 & .45 & I. 50 \\
\hline .20 & .50 & I. 75 \\
\hline $5^{\circ}$ & I $5^{\circ}$ & 500 \\
\hline .00 & 3.50 & I 200 \\
\hline .20 & .60 & 2.00 \\
\hline .20 & .45 & I. $5^{\circ}$ \\
\hline .20 & .50 & I. 75 \\
\hline .15 & .45 & $1.5^{\circ}$ \\
\hline .15 & .45 & I. $5^{0}$ \\
\hline .20 & .50 & I. 75 \\
\hline .15 & .45 & I. 50 \\
\hline .15 & .40 & I. 25 \\
\hline
\end{tabular}

Per Per Per

4 Qts. Peck Bush.

.65 I.IO 4.00

.65 I.10 4.00

.65 I.IO 4.00

$\begin{array}{lll}1.00 & \text { I.75 } & 6.50\end{array}$

.65 I.IO 4.00

$\begin{array}{lll}.60 & 1.00 & 3.75\end{array}$

.65 I.IO 4.00

.65 I.IO 4.00

.65 I.10 4.00

$\begin{array}{lll}.75 & \mathrm{I} .25 & 4.50\end{array}$

$\begin{array}{lll}.75 & 1.25 & 4.50\end{array}$

.65 I.10 4.00

.65 I.IO 4.00

.65 I.I0 4.00

.65 I.IO 4.00

$\begin{array}{lll}.60 & \mathrm{I} .00 & 3.75\end{array}$

.65 I.IO 4.00

.65 J.IO 4.00

$\begin{array}{lll}.60 & 1.00 & 3.75\end{array}$

.65 I.IO 4.00

.65 I.IO 4.00

$\begin{array}{lll}.75 & \mathrm{I} .25 & 4.50\end{array}$

$\begin{array}{lll}.60 & \text { I.oo } & 3.25\end{array}$

$\begin{array}{lll}.60 & 1.00 & 3.25\end{array}$

$\begin{array}{lll}.60 & 1.00 \quad 3.25\end{array}$

$.60 \quad 2.00$

$.60 \quad 2.00$

$.60 \quad 1.75$

.6o 1.75

$.60 \quad 2.00$

.60 2.00

$\begin{array}{ll}.60 & 2.00\end{array}$

$.60 \quad \mathrm{I} .85$

$.60 \quad 1.75$

$\begin{array}{ll}.60 & 1.75\end{array}$

$\begin{array}{ll}.60 & 1.75\end{array}$

.6o 1.85

$\begin{array}{ll}.75 & 2.50\end{array}$

$.60 \quad 1.85$

$.60 \quad 2.00$

$.60 \quad \mathrm{I} .75$

$.60 \quad 1.75$

$.60 \quad 1.75$

.6o 1.75

$.50 \quad 1.65$

$.50 \quad 1.65$
CORN SALAD.

Per Per Per
Oz. $1 / 4 \mathrm{lb}$. ib.

COLLARDS.

Georgia White Stem..... . . I5 $\quad .25 \quad .75$

Southern Blue Stem....... I5 . . . 55

Buist's Cabbage .......... .I5 .30 I.00

Carolina Buncombe ...... . . I $\quad .25 \quad .75$

\section{CRESS, or Pepper Grass.}

Curled ................ .Io .I5 .40

Water ................. .25 $\quad .75 \quad 2.75$

\section{CUCUMBER.}

Early White Spine....... . . . .25 .75

Arlington Early W. Spine. $\quad$. Io $\quad .30 \quad .85$

$\begin{array}{llll}\text { Buist's Perfection W. Spine } & \text {.I } 5 & .35 & \text { I.25 }\end{array}$

The Klondike ......... . Io $.25 \quad .85$

Early Frame ............ . IO .25 .85

Davis Perfect ........... .IO .30 1.00

Early Cluster .......... . . $\quad .25 \quad .85$

Everbearing .......... . I0 $.25 \quad .85$

London Long Green ..... . . Io $\quad .30 \quad 1.00$

Buist's Long Green ...... . . I5 $\quad .35 \quad$ I.25

Green Prolific Pickle ..... . . IO $.25 \quad .85$

Chicago Pickling ........ . . I0 $.25 \quad .85$

Jersey Pickling .......... . . IO $\quad .25 \quad .85$

Fordhook Pickling ....... .I5 .30 1.00

Gherkin, or Burr ...... .I5 .40 1.25

\section{EGG PLANT.}

Large New York Purple.. 35 I.IO 3.50

Buist's Large Purple ..... $\quad .40 \quad$ I.25 $\quad 4.25$

Black Beauty ............ .40 I.25 4.25

\section{ENDIVE.}

Green Curled ........... . . Io .30 I.00

White Curled ........... . . I0 .30 1.00

Broad Leaved ........... . . Io $\quad .30 \quad$ I.00

Moss Curled ............. . . .35 Io

\section{KALE.}

Green Curled Scotch...... .io . $20 \quad .65$

Dwarf German Greens.... . . 10 .20 $\quad .65$

Dwarf Curled Erfurt.... . . I5 $.25 \quad .90$

Curled Siberian .......... . . 10 .20 $\quad .60$

Dwarf Curled Berlin..... . . I5 $\quad .25 \quad .75$

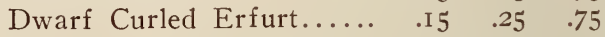

\section{KOHL RABI.}

Large Green, or White... . . I0 $\quad .30$ 1.00

Early White Vienna...... $\quad .20 \quad .50 \quad 1.75$

$\begin{array}{llll}\text { Short-Leaved W. Vienna.. } & .25 & .75 & 2.25\end{array}$

Early Purple Vienna..... $.20 \quad .60 \quad 2.00$

\section{LEEK.}

Large London Flag...... $20 \quad .45$ I.50

Buist's Mammoth .......... .20 50 I 75

$\begin{array}{llll}\text { Large Musselburgh ...... } & .20 & .50 & 1.65\end{array}$

Monstrous Carentan ..... $20 \quad .50 \quad 1.65$ 
LETTUCE.

Buist's Perfection Forcing. Early Cabbage, or Butter... Improved Big Boston .... Early Dutch Butter....... Arlington Tennisball ..... Early Boston Market...... Improved Early Hanson... Curled Simpson, blk-seed. Grand Rapids Forcing..... Early Curled Simpson..... Early Curled Silesia...... Early Prize Head.......... The Deacon ............. Lapps Silamander.

Buist's Unrivalled ............. New York Cabbage........ Buist's Hot Weatler........ All Riglit...................... Philadelphia Market ...... Improved Royal Cabbage.. Salamander ............. Paris Vhite Romaine....

\begin{tabular}{|c|c|c|}
\hline $\begin{array}{l}\text { er } \\
z z \text { z. }\end{array}$ & $\begin{array}{c}\mathrm{Per} \\
\mathrm{x} / 4 \mathrm{lb} .\end{array}$ & $\begin{array}{l}\text { Per } \\
\text { l. }\end{array}$ \\
\hline 20 & .60 & 1.7 \\
\hline & .25 & \\
\hline & & 1.2 \\
\hline & & \\
\hline & .25 & 9 \\
\hline & & \\
\hline & & \\
\hline & & 9 \\
\hline & .2 & \\
\hline .10 & .2 & 9 \\
\hline .IO & .2 & 9 \\
\hline .10 & & \\
\hline .15 & $\cdot 3$ & I.I \\
\hline .20 & .50 & 1.5 \\
\hline .20 & .50 & $I$ \\
\hline . IO & & \\
\hline 20 & .60 & 20 \\
\hline .20 & .50 & I \\
\hline .IO & 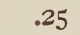 & 0 \\
\hline IO & & \\
\hline IO & .2 & \\
\hline 10 & & \\
\hline
\end{tabular}

\section{MELON-Cantaloupe.}

Perfection Rocky Ford...

Burpee's Fordhook .......

Burrell's Gem ...........

Eden Gem .............

Van Buskirk's Net'd R'k..

Rocky Ford (Buist's Selected) $\quad . . \ldots \ldots \ldots \ldots$

Emerald Gem $\ldots \ldots \ldots \ldots$.

Extra Early Hackensack..

Netted Green Citron......

Netted Nutmeg .........

Rocky Ford ............

Long Island Beauty.......

Baltimore Market ........

Early Jenny Lind ........

Improved Jenny Lind .....

Norfolk Button ..........

Miller's Cream, or Osage..

Golden Gem .............

Champion Market ........

Chicago Market ..........

New Orleans Market .....

Large Hackensack ........

Montreal Market ........

Washington Market ......

Paul Rose ...............

Banana

\section{MELON-Water.}

Tom IVatson ............ . I0 $30 \quad .90$

Kleckley's Sweet ......... Io $\quad .25 \quad .75$

Halbert Honey ............. . . . $\quad .25 \quad .75$

Florida Favorite ..........

Augusta Rattlesnake ......

Dixie ..................

Monte Cristo .............

Alabama Sweet ...........

Blue Gem ...............

Sweet Heart ..............

Carolina Bradford .........

Ice Cream ...............

Light Icing...$\ldots \ldots \ldots$.

Dark Icing
MELON-Water Con't

Per Per Per

Jordan's Gray Monarch.... . . I0 $2.5 \quad .75$

Mlountain Sweet ......... 10 . 10 .20

Duke Jones ............ . .

Kolb Gem .............. . . .

Premium Triumph ......

Cuban Queen ........... . . I0 .20 .60

\section{MUSTARD.}

Mammoth Southern Curled .IO .20 .50

$\begin{array}{llll}\text { Nammoth Ostrich Plume.. } & \text {. I5 } & .20 & .55\end{array}$

Southern Creole .......... . . IO .20 $\quad .50$

White London ........... .05 .I0 .25

Black, or Brown ........ . . 05 .IO .25

\section{MUSHROOM SPAWN.}

English, in double bricks, Imported. Price I5 cts. each; I2 bricks.......... Ioo pounds $\ldots \ldots \ldots \ldots$..... 50 " I.5C 7.00 3.50 2.00

\section{OKRA, or Gumbo.}

Buist's Dwarf Prolific..... .I0 .I5 $\quad .45$

Tall, or Long Green .......

Perkins' Mammoth Podded . I0 $\quad .20 \quad .50$

White Velvet ............ .IO .I5 .40

\section{ONION.}

Buist's Yel. G'be Danvers. .I5 .4O

Yellow Globe Danvers.... . I5 .35 I. IO

Yellow Danvers .......... . I5 .35 I. Io

Large Red Wethersfield... $\quad$. I5 $\quad .40$ I 25

Yellow Dutch, or Strasb'g. .I5 .35 I IO

White, or Silver Skin.... $20 \quad 60 \quad 2.00$

Southport Yellow Globe.. .I5 .40 I.20

Southport Red Globe...... I5 4 I5 1.30

$\begin{array}{llll}\text { Southport White Globe.... } & 25 & .75 & 2.25\end{array}$

Australian Brown ........ . . . .40 I. IO

Extra Early Red ......... . I5 .40 I.IO

Giant 'Yellow Prizetaker.. .I5 .40 I.IO

ONION-Italian varieties.

Red Bermuda (Teneriffe)

White Bermuda (do.) ....

Crystal White (do) ......

Mammoth Silver King....

Mammoth Red Victoria...

Mammoth White Victoria

Extra Early White Pearl..

Extra Early White Queen.

Early White Barletta .....

\section{ONION SETS.}

Yellow Onion Sets ....... $.12 \quad .65 \quad 2.25$

Dark Red Onion Sets.....

White Onion Sets ........

\section{PARSLEY.}

Double Curled ........... IO $\quad .25 \quad .75$

Buist's Garnishing ........... . .

Dark Moss Curled ......... . Io $\quad .25 \quad .75$

$\begin{array}{llll}\text { Dwarf Extra Curled ..... . . I0 } & .25 & .75\end{array}$

Plain, or Single ......... . Io $\quad .25 \quad .75$ 


\begin{tabular}{|c|c|c|c|}
\hline ARS & $\begin{array}{l}\text { Per } \\
\text { Oz. }\end{array}$ & $\begin{array}{l}\text { Per } \\
1 / 4 \mathrm{lb} \text {. }\end{array}$ & lb. \\
\hline or Hollow Crown.. & . 10 & .20 & \\
\hline .......... & .10 & .20 & \\
\hline PEPPER. & & & \\
\hline $\mathrm{Be}$ & .20 & .50 & \\
\hline . & .20 & .50 & \\
\hline K & .20 & .60 & \\
\hline G & .40 & I. 25 & \\
\hline $\mathrm{E}$ & .25 & .65 & \\
\hline $\mathrm{Re}$ & .20 & .60 & \\
\hline Small Rec & .25 & .75 & \\
\hline
\end{tabular}

POTATOES. For Prices, see inside Cover Page. PUMPKIN.

Mammoth Gold. Cashaw.. .Io $.20 \quad .65$ Green Striped Cashaw.... Yel. Cashaw, or C'k N'k.. Large Cheese ........... $\begin{array}{llll}\text { Quaker Pie ............. } & \text {. I0 } & .25 & .65\end{array}$ $\begin{array}{llll}\text { Mammoth King .......... } & .20 & .40 & \text { I.25 }\end{array}$ Connecticut Field ........ . I0 . I5 .40 Mammoth Field .......... Mammoth Etamps ........ . I5 30 1.00

\section{RADISH.}

Buist's Sel. Long Scarlet. . . I5 $.20 \quad 65$ Early Long Scarlet....... $\begin{array}{llll}\text { Long Brightest Scarlet .... } & \text {.10 } & .20 & .60\end{array}$ Cincinnati Market ........ . . 10 .20 60 Buist's Early Lg. White.. . I0 $\quad .25 \quad .75$ Long White Icicle ....... . . . Long White Vienna ...... . . I0 $.20 \quad .50$ Early Deep Scarlet Tur.... $.10 \quad .20 \quad .50$ Scar. Turnip, W.-Tipped.. . I0 $\quad .20 \quad .50$ Early Erfurt Scar. Turnip. $\quad$.IO $\quad .20 \quad .50$ Buist's Cardinal Globe...... $\quad$. I0 $\quad .25 \quad .75$ $\begin{array}{llll}\text { Prussian Scarlet Globe.... } & \text {.I5 } & .25 & .75\end{array}$ $\begin{array}{llll}\text { Lightning Scarlet Forc'g... } & \text {.IO } & .20 & .65\end{array}$ Buist's Scarlet Button..... . . I0 $\quad .20 \quad .65$ Scarlet French Breakfast.. .10 $.20 \quad .60$ Early Scar. Olive-shaped.. Early Half-Long Scarlet.. Early White Turnip...... . . 10 .20 60 Early White Box........ . . 10 .20 60 Improved Chartier ......... . . 10 . 20 .60 White Summer Strasburg. 10 . 20 .60 Celestial, or White Chinese. $\quad$.I $\quad .35$ I.00 Chinese Rose ............ . . I0 $\quad .30 \quad .75$ Round Scarlet China ..... Round Black Spanish...... . . 10 o $20 \quad .60$ $\begin{array}{llll}\text { White Summer Turnip.... } & \text {. I } & .25 & .65\end{array}$ Yellow Summer Turnip... . . 10 . 25 .65 RHUBARB.
SPINACH. Contiuued. Monstrous Viroflay ....... Enkhuizen Long Standing. Victoria ................

\section{SQUASH.}

Early White Bush ....... . . . .20 .60 Extra Early White Bush. . I0 $.20 \quad .60$ Mammoth White Bush.... .I0 $.20 \quad .60$ Yellow Sum. Crook Neck.. Giant Summer C'k Neck.. Boston Marrow .......... . . . 20 . 60 Buist's Improved Marrow. $\quad .20 \quad .25 \quad .80$ Hubbard ............... . .

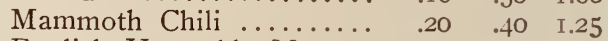
English Vegetable Marrow .I5 .30 1.00

\section{TOMATO.}

Buist's Beauty .......... $.25 \quad .75 \quad 2.50$ Buist's Belle ............. $\quad .35 \quad .85 \quad 3.00$ Buist's Majestic .......... .40 I.IO 3.50 Buist's Prize Stone ...... $\quad .30 \quad .75 \quad 2.50$ Buist's New Ideal............ $\quad .50 \quad$ I.25 4.00 $\begin{array}{lllll}\text { The Success } \ldots \ldots \ldots \ldots . & .25 & .75 & 2.50\end{array}$ $\begin{array}{llll}\text { Livingstone's Perfection.. } & .20 & .50 & 1.75\end{array}$ Livingstone's Beauty ...... $.20 \quad .50$ I.75 Livingstone's Stone $\ldots . . . . \quad .20 \quad .50 \quad$ I.75 $\begin{array}{llll}\text { Livingstone's Globe ....... } & .30 & .85 & 3.00\end{array}$ $\begin{array}{llll}\text { June Pink } \ldots \ldots \ldots \ldots \ldots & .30 & .85 & 2.75\end{array}$

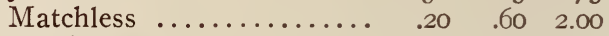
$\begin{array}{lllll}\text { Ponderosa } \ldots \ldots \ldots \ldots . . .6 & .30 & .75 & 2.75\end{array}$ $\begin{array}{llll}\text { Spark's' Earliana .......... } & .25 & .65 & 2.50\end{array}$ $\begin{array}{lllll}\text { Chalk's Early Jewel ...... } & .30 & .75 & 2.75\end{array}$ Buist's Selected Trophy... $.20 \quad .50$ I.75 Buist's Selected Paragon.. $\quad .20 \quad .50$ I.75 Buist's Selected Acme.... .20 .50 I.7.5 $\begin{array}{lllll}\text { Dwarf Stone } \ldots \ldots \ldots \ldots & .25 & .75 & 2.50\end{array}$ Bonnie Best $\ldots \ldots \ldots \ldots \ldots . .25 \quad .75 \quad 2.50$ $\begin{array}{llll}\text { Dwarf Champion .......... } & .25 & .60 & 2.00\end{array}$ Golden Trophy .......... $\quad .25 \quad .75 \quad 2.25$ Yellow Plum ........... $\quad .25 \quad .75 \quad 2.25$

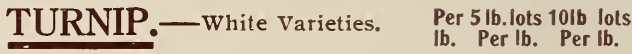

$\begin{array}{llll}\text { Red or Purple Top........ } & .50 & .40 & .35\end{array}$ Early White Flat Dutch.... $\quad .50 \quad .40 \quad .35$ Early Red Top Globe.... $\quad .60 \quad .50 \quad .40$ Buist's Pur. Top Globe... $\quad .60 \quad .50 \quad$. 50 $\begin{array}{llll}\text { Large White Globe........ } & .50 & .40 & .35\end{array}$ $\begin{array}{llll}\text { Large White Norfolk...... } & .50 & .40 & .35\end{array}$ $\begin{array}{llll}\text { Pomeranean White Globe. } & .50 & .40 & .35\end{array}$ Snow White Globe....... $\quad .50 \quad .40 \quad .35$ Early White Egg......... $50 \quad .50 \quad .40 \quad .35$ Large Cow Horn.......... $\quad .50 \quad .40 \quad .35$ $\begin{array}{llll}\text { Seven Top (for Greens).. } & .50 & .40 & .35\end{array}$ $\begin{array}{llll}\text { Southern Prize (do.)...... } & .50 & .40 & .35\end{array}$

TURNIP_Yellow varieties.

Yellow Aberdeen ........ $\quad .50 \quad .40 \quad .35$ $\begin{array}{llll}\text { Yellow Stone } \ldots \ldots \ldots \ldots & .50 & .40 & .35 \\ & .40 & .35\end{array}$ Golden Ball ............ $\quad .50 \quad .40 \quad .35$ Amber Globe $\ldots \ldots \ldots \ldots \ldots .6 \%$ Yellow Globe ............ $\quad .50 \quad .40 \quad .35$

\section{TURNIP, RUTA BAGA,}

Pur. Top Yel. Ruta Baga.. Buist's Yellow Ruta Baga. Long Island Swede ...... Large White, or Russian.. Breadstone, or Budlong...
$.50 \quad .40$ $.60 \quad .50$ $.50 \quad .40$ $.60 \quad .50$ $.60 \quad .50$ 

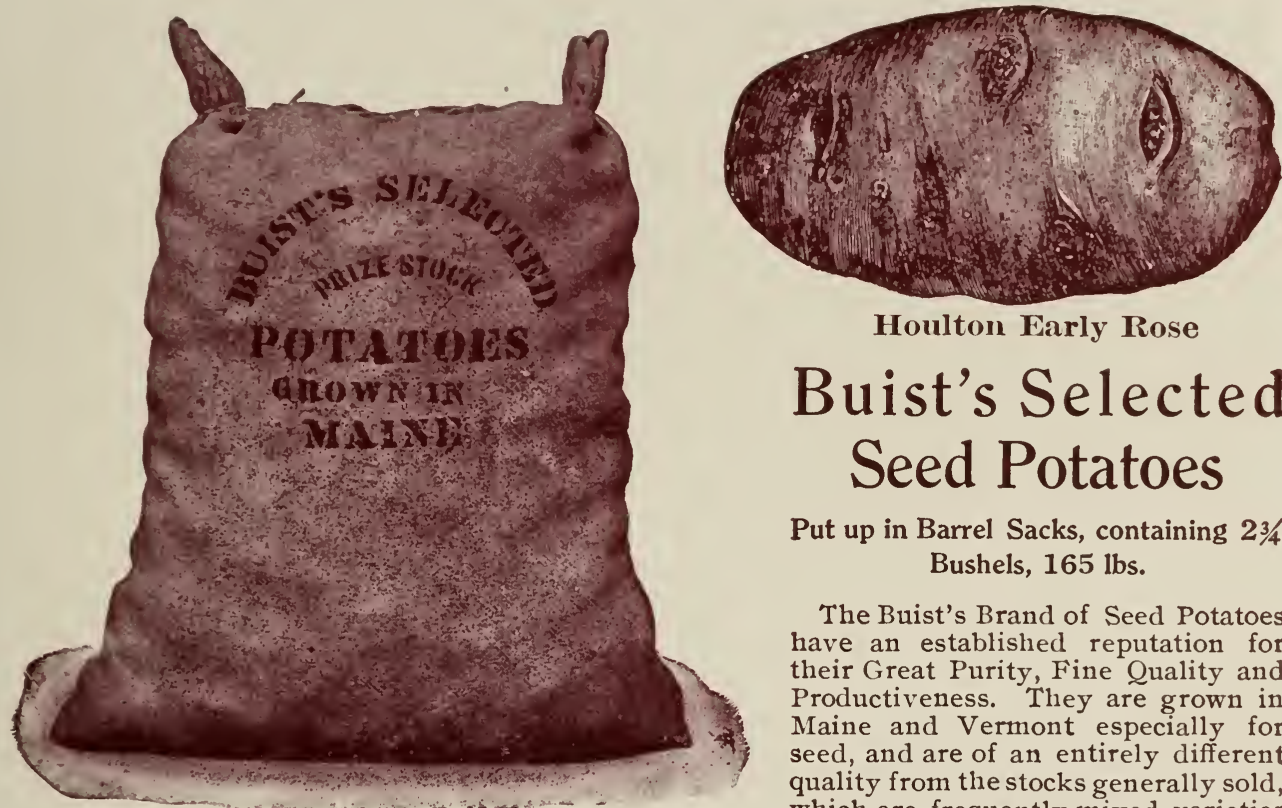

Houlton Early Rose

\section{Buist's Selected Seed Potatoes}

Put up in Barrel Sacks, containing 23/4 Bushels, 165 lbs.

The Buist's Brand of Seed Potatoes have an established reputation for their Great Purity, Fine Quality and Productiveness. They are grown in Maine and Vermont especially for seed, and are of an entirely different quality from the stocks generally sold, which are frequently mixed varieties of a very inferior grade, with the name branded to suit the order. Our Quotations are Subject to Market Changes.

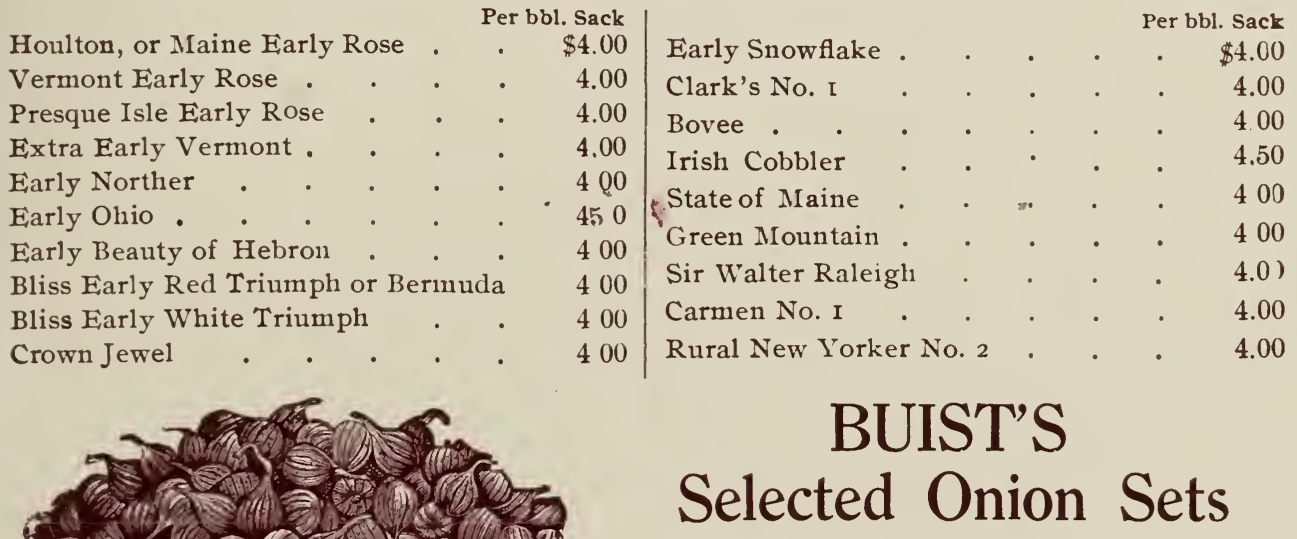

Our Onion Sets are very bright, handsome and well-cleaned samples, and of small size, which are the best for planting. They are far superior to Western or Sonthern-grown sets, which are generally large in size and spongy, sprouting earlier and shooting to seed before forming large onions.

\section{YELLOW ONION SETS}

Per qt., 12 cts. ; pk., 65 cts. ; bus., $\$ 2.25$; 5 bus. at $\$ 2.00$.

\section{DARK RED ONION SETS}

Per qt., 12 cts. ; pk., 75 cts. ; bus., $\$ 2.50$; 5 bus. at $\$ 2.25$.

\section{WHITE ONION SETS}

Per qt., 15 cts. ; pk., 85 cts. ; bus., $\$ 3.00$; 5 bus. at $\$ 2.75$. 

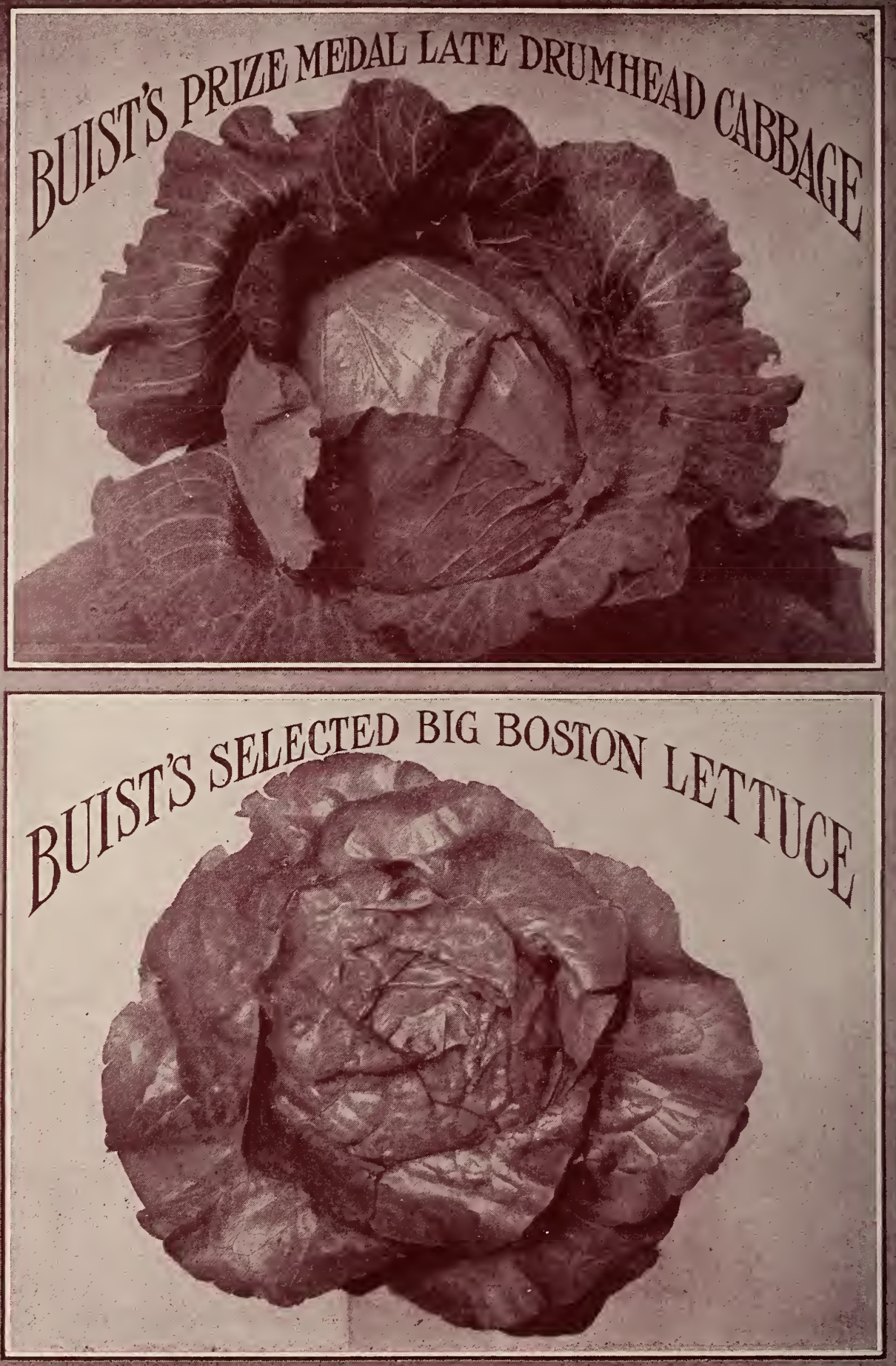

\section{ROBERT BUIST COMPANY S PHILADELPHIA

